\title{
Fourier analysis of an equal-order incompressible flow solver stabilized by pressure gradient projection
}

\author{
Gustavo C. Buscaglia ${ }^{\mathrm{a}, * 1}$, Fernando G. Basombrío ${ }^{\mathrm{a}}$ and Ramon Codina ${ }^{\mathrm{b}}$ \\ ${ }^{a}$ Grupo Mecánica Computacional, Centro Atómico Bariloche and Instituto Balseiro, 8400 Bariloche, Argentina \\ ${ }^{\mathrm{b}}$ Escola Tècnica Superior d'Enginyers de Camins, Canals i Ports, Universitat Politècnica de Catalunya, \\ Jordi Girona 1-3, Edifici C1, 08034 Barcelona, Spain
}

\begin{abstract}
SUMMARY
Fourier analysis techniques are applied to the stabilized finite element method (FEM) recently proposed by Codina and Blasco for the approximation of the incompressible Navier-Stokes equations, here denoted by pressure gradient projection (SPGP) method. The analysis is motivated by spurious waves that pollute the computed pressure in start-up flow simulation. An example of this spurious phenomenon is reported. It is shown that Fourier techniques can predict the numerical behaviour of stabilized methods with remarkable accuracy, even though the original Navier-Stokes setting must be significantly simplified to apply them. In the steady state case, good estimates for the stabilization parameters are obtained. In the transient case, spurious long waves are shown to be persistent when the element Reynolds number is large and the Courant number is small. This can be avoided by treating the pressure gradient projection implicitly, though this implies additional computing effort. Standard extrapolation variants are unfortunately unstable. Comparisons with Galerkin-least-squares (GLS) method and Chorin's projection method are also addressed. Copyright (C) 2000 John Wiley \& Sons, Ltd.
\end{abstract}

KEY WORDS: incompressible flow; von Neumann's analysis; spurious pressure wave; stabilized methods

\section{INTRODUCTION}

Over the last few years a lot of effort has been devoted to the development of finite element methods (FEMs) for incompressible flows allowing for the identical interpolation for velocity and pressure unknowns to be used. It is well known that the usual Galerkin formulation violates the Babuška-Brezzi (BB) stability condition for such equal-order approximations, so that stabilization is needed. Several stabilized formulations have been developed over the years. Some of them, such as the popular Galerkin-least-squares (GLS) method [1,2], explicitly perturb the Galerkin formulation by mesh-dependent terms so as to improve stability. In other

\footnotetext{
* Correspondence to: Grupo Mecánica Computacional, Centro Atómico Bariloche and Instituto Balseiro, 8400 Bariloche, Argentina.

${ }^{1}$ E-mail: gustavo@cab.cnea.gov.ar
} 
formulations, the stabilization terms are implicit within a fractional step algorithm. This is the case, for example, of projection methods based on the early ideas of Chorin [3] and Temam [4] (see, e.g. References [5-11]).

The equal-order method analysed in this article combines, in some sense, the two stabilization procedures mentioned above. An explicit stabilization term is incorporated, which in turn mimics the effect of fractional step methods. To our knowledge, the first precedent of this method was proposed by Habashi et al. [12] as the following modification of the zerodivergence constraint:

$$
\operatorname{div} u-\lambda \nabla^{2} p=-\lambda \operatorname{div} g
$$

where $g=\nabla p$ and $\lambda$ is a small parameter. Although at the continuous level the terms containing $\lambda$ cancel exactly, upon finite element discretization, cancellation no longer occurs and these terms in fact stabilize the formulation. Equivalent terms in the finite element equations were later identified by Zienkiewicz and Codina [13] as explaining the good behaviour of an equal-order fractional step method (in their scheme, $\lambda$ was in fact the time step). Finally, Codina and Blasco [14-16] formulated and analysed theoretically an equalorder method based on these ideas. From the convergence analysis, an elementwise estimate $\lambda \sim h^{2}$ (for $h$ being small) was derived, and optimal convergence rates obtained. We will refer to this method hereafter as the stabilized by pressure gradient projection (SPGP) method. Recent work by Codina [17] has shown a link between this method and the sub-grid scales (SGS) method [18].

From the computational point of view, the main drawback of the SPGP method is the introduction of the projection of the pressure gradient as a new unknown of the problem, thus
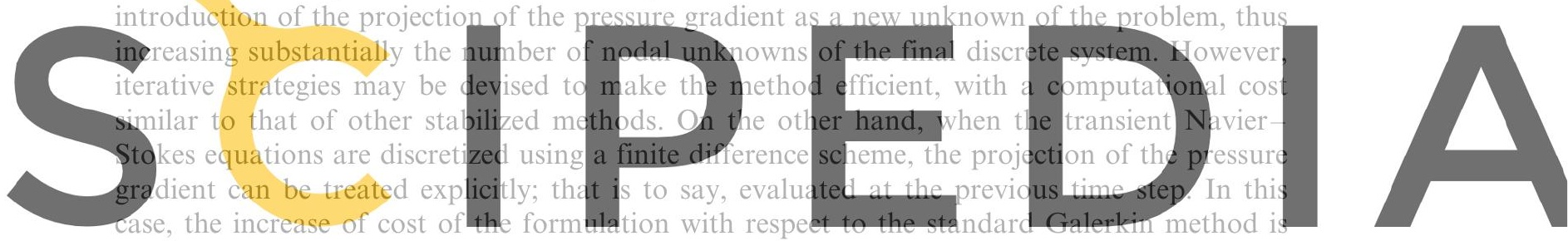

very low. First, a stabilization matrix must be built up, and at the end of each time step the

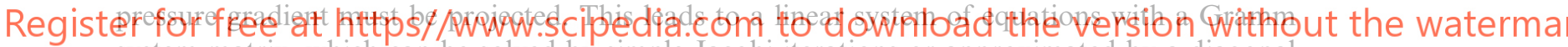
system matrix, which can be solved by simple Jacobi iterations or approximated by a diagonal system (lumped form). The number of unknowns is not increased.

Many numerical tests have recently been performed to the SPGP method, involving steady and transient, two- and three-dimensional flows [Buscaglia G, Carrica P. Unpublished results; 19], with quite good results. A turbulent code based on the SPGP method also exhibits good behaviour [Lew A, Buscaglia G, Carrica P. A robust equal-order finite element formulation for the k-epsilon turbulence model. Submitted]. Most of these tests deal with the pressure gradient projection explicitly. Comparing the SPGP and GLS methods, it was found that the former leads to better conditioning of the system matrix and smoother temporal behaviour of the pressure field in transients. As a drawback, spurious long-wave pressure transients in strongly accelerated flows (such as start-up flows) were detected. One of these cases is reported below. These spurious transients do not affect the velocity field, but render the pressure field useless 
until their extinction. Fortunately, no such phenomenon occurs in smooth flows (such as vortex shedding flows).

Summarizing, its overall performance makes the SPGP method attractive for equal-order finite element treatment of incompressible flows, mainly transient ones, and further work is needed to understand and improve its properties. Stabilized methods are prone to introduce spurious pressure wave perturbations. This is a price to be paid for avoiding the BB condition.

In this paper, Fourier analysis techniques are applied to the SPGP method and some of its variants. Several simplifications are introduced to render this analysis feasible. First, a one-dimensional model problem that mimics the Navier-Stokes equations is introduced and discretized. Second, the domain is assumed to be $(-\infty,+\infty)$, so that all nodes are equivalent. Finally, the convective non-linear term is linearized. From the Fourier analysis of the discrete equations, appropriate choices of stabilization coefficients are obtained. In the transient case (classical von Neumann's analysis) stability is discussed. Moreover, a spurious oscillatory behaviour is identified, which explains (both qualitatively and quantitatively) two-dimensional numerical results for start-up flow around a circular cylinder at a Reynolds number of 3000. In particular, it is shown that the explicit treatment of the pressure gradient projection activates this spurious behaviour and that a high-Reynolds number stabilization coefficient improves the results. Comparisons with the GLS and Chorin's methods are also addressed. Numerical results of the one-dimensional model problem in a bounded domain without linearizing the convective term show that the conclusions from Fourier analysis apply in more realistic situations.
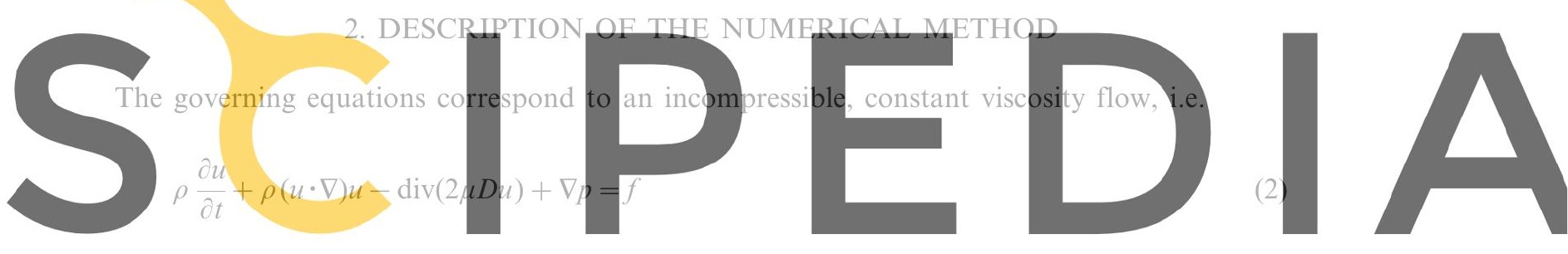

Register for free at https//www.scipedia.com to download the version without the watermar

where $u$ is the velocity field; $\rho$ is the density; $\mu$ is the dynamic viscosity; $D$ is the symmetric gradient operator, i.e. $(D u)_{i j}=\left(u_{i, j}+u_{j, i}\right) / 2 ; p$ is the pressure; and $f$ is the volumetric forces. These equations are assumed to hold in a bounded domain $\Omega$, with initial solenoidal conditions for $u$ in $\Omega$, imposed velocities on the Dirichlet boundary $\Gamma_{\mathrm{D}}$, and imposed tractions on the Neumann boundary $\Gamma_{\mathrm{N}}\left(\overline{\Gamma_{\mathrm{D}} \cup \Gamma_{\mathrm{N}}}=\partial \Omega, \Gamma_{\mathrm{D}} \cap \Gamma_{\mathrm{N}}=\varnothing\right)$.

$$
\begin{aligned}
& u(x, 0)=u_{0}(x), \quad x \in \Omega \\
& u(x, t)=g(x, t), \quad x \in \Gamma_{\mathrm{D}} \\
& (-p \mathbf{1}+2 \mu D u) \cdot \mathbf{n}=\mathscr{F}, \quad x \in \Gamma_{\mathrm{N}}
\end{aligned}
$$


Now, let $\mathscr{T}_{h}$ be a finite element partition of $\Omega$, and let $V_{h} \subset H^{1}(\Omega)^{n_{\mathrm{sd}}}$ be an associated finite element space to approximate the velocity field, where $n_{\mathrm{sd}}$ is the number of space dimensions. We assume that $V_{h}$ consists of piecewise linear, bilinear or trilinear vector fields. We define, as usual,

$$
\begin{aligned}
& V_{h \mathrm{D}}=\left\{v_{h} \in V_{h}, v_{h}=g \text { on } \Gamma_{\mathrm{D}}\right\} \\
& V_{h 0}=\left\{v_{h} \in V_{h}, v_{h}=0 \text { on } \Gamma_{\mathrm{D}}\right\}
\end{aligned}
$$

and let $Q_{h} \subset L^{2}(\Omega)$ be a finite element space for the pressure. Of most interest to us is the case when the interpolants for the pressure coincide with those used for each component of the velocity field. Finally, let $G_{h}$ be another vector finite element space, which we take to be generally coincident with $V_{h}$ (no boundary conditions imposed). The SPGP method considered thus reads [15]

\section{Find $\left(u_{h}^{n}, p_{h}^{n}, g_{h}^{n}\right) \in V_{h \mathrm{D}} \times Q_{h} \times G_{h}$ such that}

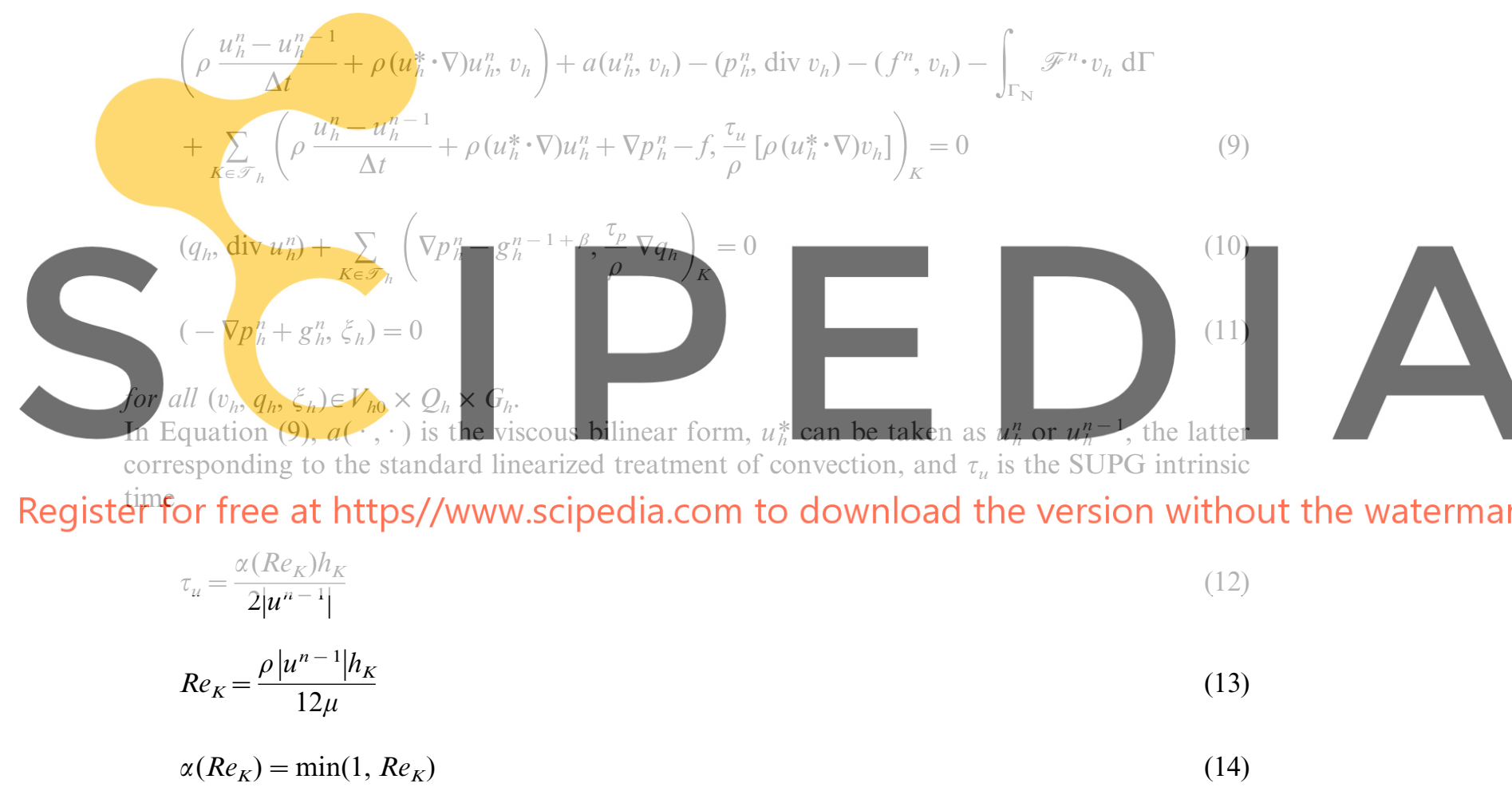

and $\tau_{p}$ is a second intrinsic time. In Reference [15], the value $\rho h_{K}^{2} /(12 \mu)$, differing just by a factor of two from the low-Reynolds number formula for $\tau_{u}$, is adopted. In practice, this value is slightly too large for high-Reynolds number computations, a better choice being $\tau_{p}=\tau_{u}$. 
Simpler formulae for the stabilization parameters, justified by a convergence analysis, were recently proposed by Codina [20]. Notice that the previous formulation is of the backward Euler kind, except for the appearance of $g_{h}^{n-1+\beta}$ if $\beta<1$. For the algorithmic cost to be competitive, the choice $\beta=0$ is mandatory.

The matrix formulation of Equations (9)-(11) is

$$
\begin{aligned}
& \frac{1}{\Delta t} \mathbf{M}_{U}\left(\underline{U}^{n}-\underline{U}^{n-1}\right)+(\mathbf{A}+\mathbf{K}) \underline{U}^{n}-\tilde{\mathbf{B}} \underline{P}^{n}=\underline{F} \\
& \mathbf{B}^{T} \underline{U}^{n}+\frac{1}{\rho} \mathbf{L} \underline{P}^{n}-\frac{1}{\rho} \mathbf{D}^{T} \underline{G}^{n-1+\beta}=\underline{0} \\
& -\mathbf{C} \underline{P}^{n}+\mathbf{M} \underline{G}^{n}=\underline{0}
\end{aligned}
$$

where $\underline{U}^{n}, \underline{P}^{n}$ and $\underline{G}^{n}$ are the vectors containing the unknowns, $\mathbf{M}_{U}, \mathbf{A}$ and $\mathbf{K}$ are the matrices arising from the temporal derivative, convective and viscous terms respectively (including the stabilization terms), and

$$
\mathbf{B}_{I J}=\int_{\Omega} M^{J} \operatorname{div} N^{I} \mathrm{~d} \Omega
$$
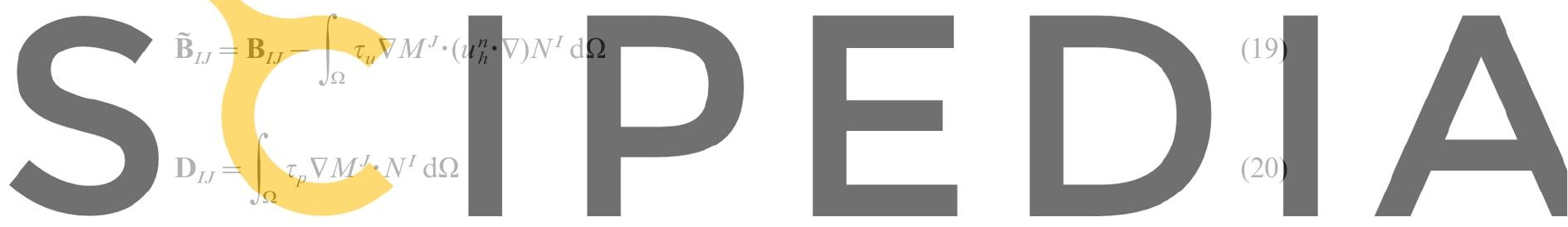

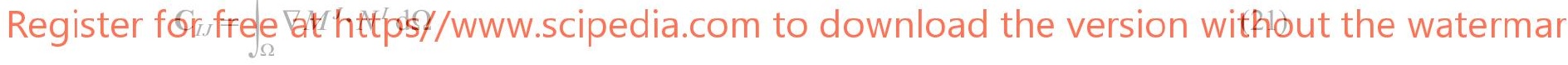

$$
\begin{aligned}
& \mathbf{L}_{I J}=\int_{\Omega} \tau_{p} \nabla M^{J} \cdot \nabla M^{I} \mathrm{~d} \Omega \\
& \mathbf{M}_{I J}=\int_{\Omega} M^{J} M^{I} \mathrm{~d} \Omega
\end{aligned}
$$

$M^{I}$ and $N^{I}$ being respectively, the $I$ th basis function for pressure and velocity ( $N^{I}$ is, thus, vectorial). The mass matrix $\mathbf{M}$ can be used in consistent form or lumped form. No significant difference was found using linear elements, so that the lumped form is adopted. 


\section{FOURIER ANALYSIS OF THE STEADY STATE}

To perform the Fourier analysis, a model one-dimensional problem is introduced that retains the main characteristics of the Navier-Stokes equations. We keep the notation $u$ and $p$ for the unknowns to make the analogy evident. The proposed equations are

$$
\begin{aligned}
& \rho \frac{\partial u}{\partial t}+\rho u \frac{\partial u}{\partial x}-2 \mu \frac{\partial^{2} u}{\partial x^{2}}+\frac{\partial p}{\partial x}=f \\
& \frac{\partial u}{\partial x}=0
\end{aligned}
$$

Let us begin omitting the SUPG terms $\left(\tau_{u}=0\right)$. Let us also linearize the problem replacing the non-linear term $\rho u(\partial u / \partial x)$ by $\rho c(\partial u / \partial x)$. The SPGP method, with M lumped and assuming $f$ is continuous and piecewise linear, leads to the following stencil:

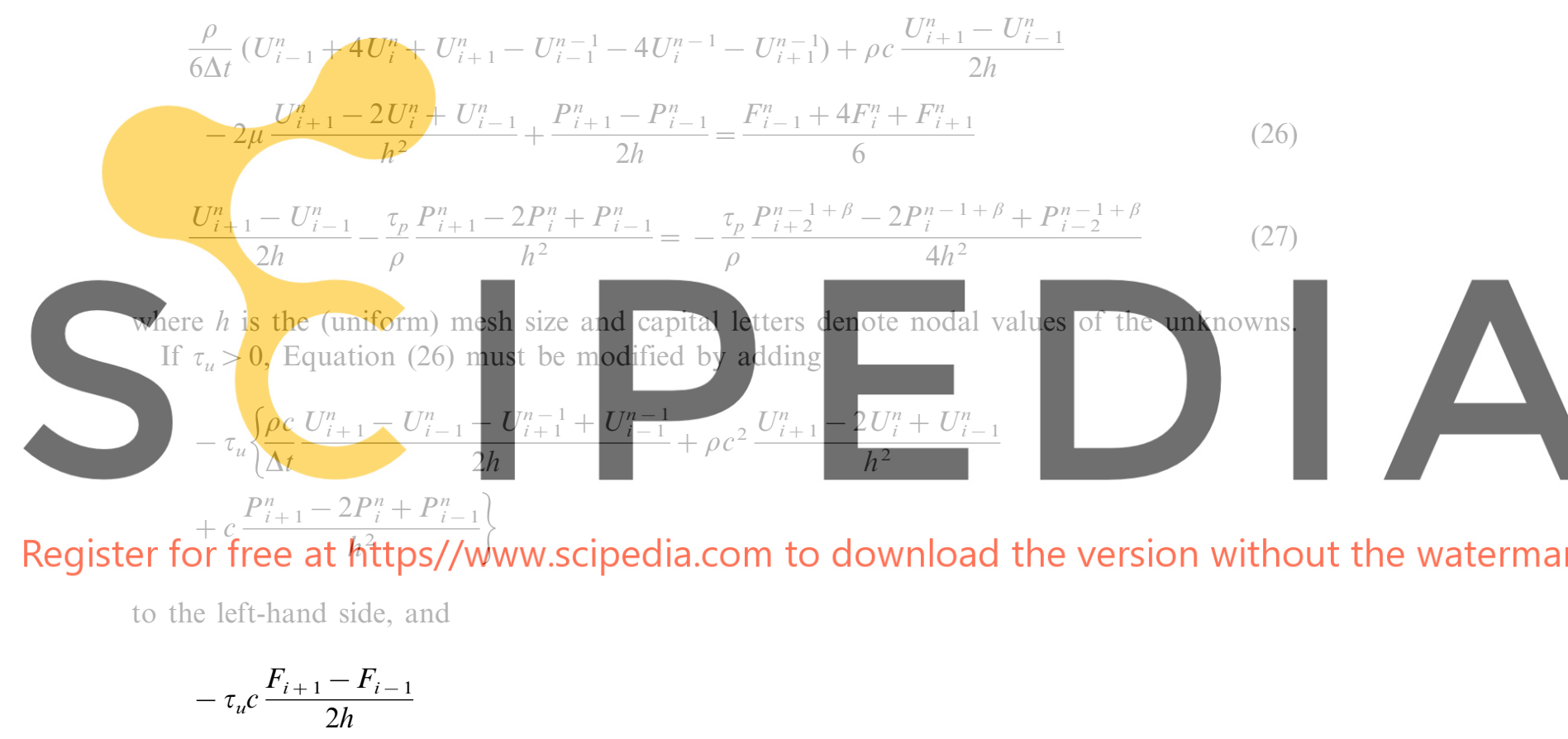

to the right-hand side.

The Fourier analysis of the steady state, which is restricted to Stokes flow $(\rho=0)$, roughly follows the lines of Reference [21]. Let us consider the following stencil:

$$
-(2 \mu+v) \frac{U_{i+1}-2 U_{i}+U_{i-1}}{h^{2}}+\frac{P_{i+1}-P_{i-1}}{2 h}=\frac{F_{i-1}+F_{i}+F_{i+1}}{6}
$$




$$
\frac{U_{i+1}-U_{i-1}}{2 h}-\alpha \frac{P_{i+1}-2 P_{i}+P_{i-1}}{h^{2}}+\gamma \frac{P_{i+2}-2 P_{i}+P_{i-2}}{4 h^{2}}=-\delta \frac{F_{i+1}-F_{i-1}}{2 h}
$$

This stencil, with suitable values for the constants $v, \alpha, \gamma$ and $\delta$, corresponds to the steady state formulation of the SPGP method $\left(U_{i}^{n}=U_{i}^{n-1}=U_{i}, P_{i}^{n}=P_{i}^{n-1}=P_{i}\right)$. It also allows for the comparison of the SPGP method $(\delta=0, \alpha=\gamma)$ with the GLS method $(\delta=\alpha, \gamma=0)$. Now, let

$$
\mu^{\prime}=2 \mu+v, \quad \tilde{\alpha}=\frac{\alpha \mu^{\prime}}{h^{2}}, \quad \tilde{\gamma}=\frac{\gamma \mu^{\prime}}{h^{2}}, \quad \tilde{\delta}=\frac{\delta \mu^{\prime}}{h^{2}}
$$

and defining

$$
V=\frac{U \mu^{\prime}}{h^{2}}, \quad G_{i+1 / 2}=\frac{P_{i+1}-P_{i}}{h}
$$

the stencil becomes

$$
\begin{aligned}
& -\left(V_{i+1}-2 V_{i}+V_{i-1}\right)+\frac{1}{2}\left(G_{i+1 / 2}+G_{i-1 / 2}\right)=\frac{F_{i+1}+4 F_{i}+F_{i-1}}{6} \\
& \frac{V_{i+1}-V_{i-1}}{2}-\tilde{\alpha}\left(G_{i+1 / 2}-G_{i-1 / 2}\right)+\frac{\tilde{\gamma}}{4}\left(G_{i+3 / 2}+G_{i+1 / 2}-G_{i-1 / 2}-G_{i-3 / 2}\right) \\
& =-\frac{\tilde{\delta}}{2}\left(F_{i+1}-F_{i-1}\right)
\end{aligned}
$$

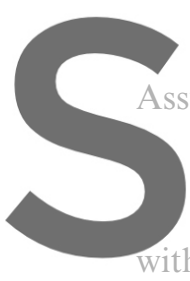

Assuming now that $i$ runs
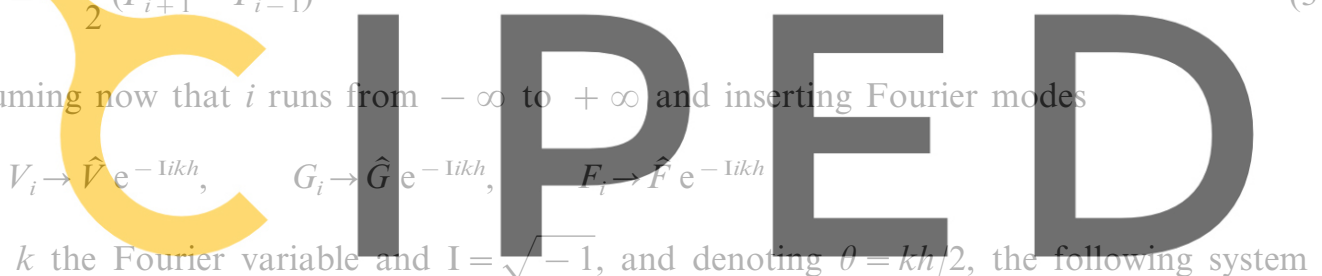

(31)

obtained:

Register for free at https//www.scipedia.com to download the version without the watermar

$$
\begin{aligned}
& 4 \sin ^{2} \theta \hat{V}+\cos \theta \hat{G}=\left(1-\frac{2}{3} \sin ^{2} \theta\right) \hat{F} \\
& \cos \theta \hat{V}+\left(\tilde{\gamma} \cos ^{2} \theta-\tilde{\alpha}\right) \hat{G}=-\tilde{\delta} \cos \theta \hat{F}
\end{aligned}
$$

Symbolic manipulation [20] was used to get $\hat{V}$ and $\hat{G}$. Its expansions around $\theta=0$ read

$$
\begin{aligned}
& S_{V}:=\frac{\hat{V}}{\hat{F}}=\tilde{\alpha}-\tilde{\delta}-\tilde{\gamma}+\left[-4 \tilde{\alpha}^{2}-\frac{2}{3} \tilde{\gamma}(-1+6 \tilde{\delta}+6 \tilde{\gamma})+\tilde{\alpha}\left(\frac{1}{3}+4 \tilde{\delta}+8 \tilde{\gamma}\right)\right] \theta^{2}+O\left(\theta^{4}\right) \\
& S_{G}:=\frac{\hat{G}}{\hat{F}}=1+\left[-\frac{1}{6}+4(-\tilde{\alpha}+\tilde{\delta}+\tilde{\gamma})\right] \theta^{2}+O\left(\theta^{4}\right)
\end{aligned}
$$


Notice that the exact dependencies (if $\theta \neq 0$ ) are $\hat{V}=0, \hat{G}=\hat{F}$, or, in other terms, $S_{V}=0$ and $S_{G}=1$. For the scheme to coincide with these exact expressions at $\theta \rightarrow 0$, the only condition is

$$
\tilde{\alpha}-\tilde{\gamma}-\tilde{\delta}=0
$$

Both the GLS and SPGP methods satisfy this condition. Let us impose thus $\tilde{\gamma}=\tilde{\alpha}-\tilde{\delta}$. In this case

$$
\begin{aligned}
& S_{V}=\left(\tilde{\alpha}-\frac{2}{3} \tilde{\delta}\right) \theta^{2}+O\left(\theta^{4}\right) \\
& S_{G}=1-\frac{\theta^{2}}{6}+O\left(\theta^{4}\right)
\end{aligned}
$$

From this we learn, on the one hand, that asymptotic accuracy (as $k h \rightarrow 0$ ) cannot be improved beyond second order (which is the expected spatial accuracy), as $S_{\mathrm{G}}$ is second-order accurate for any choice of $\tilde{\alpha}$ and $\tilde{\delta}$. On the other hand, accuracy in the velocity could be improved by choosing a linear combination of the GLS and SPGP methods, namely $\tilde{\alpha}=\frac{2}{3} \tilde{\delta}$ (implying $\left.\tilde{\gamma}=-\frac{1}{3} \tilde{\delta}\right)$. The gain is, however, not significant, as, in general, accuracy in velocity is much higher than in pressure.

The selection of $\tilde{\alpha}$ and $\tilde{\delta}$ must be made examining the accuracy when $k h$ is far from zero, as the behaviour in the vicinity of zero does not depend on these parameters. We have focused on two cases: $\operatorname{GLS}(\tilde{\alpha}=r, \tilde{\delta}=r, \tilde{\gamma}=0)$ and $\operatorname{SPGP}(\tilde{\alpha}=r, \tilde{\gamma}=r, \tilde{\delta}=0)$. Comparison is made for

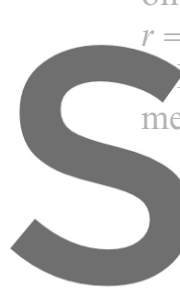
$k=10^{-1}, 10^{-2}$ and $10^{-3}$. Plots of $S_{G}$ versus $\theta$ can be seen in Figures 1 and 2

Py dircet inspection of $\mathrm{F}$

methods, $r=10^{-2}$ is the bost choice.
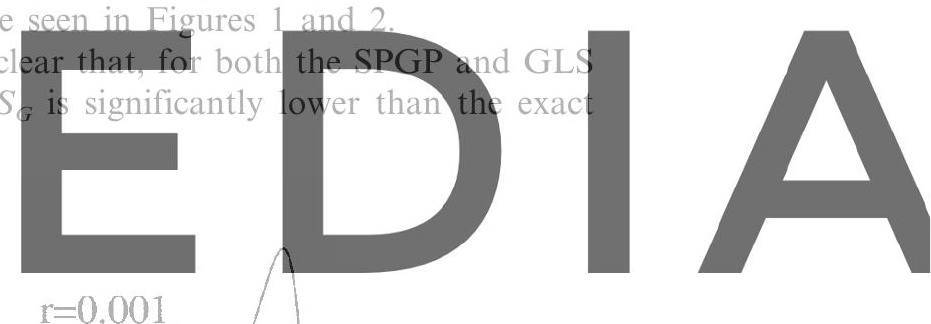

Register for free at https//www.scipedia.com to download/the version without the watermar

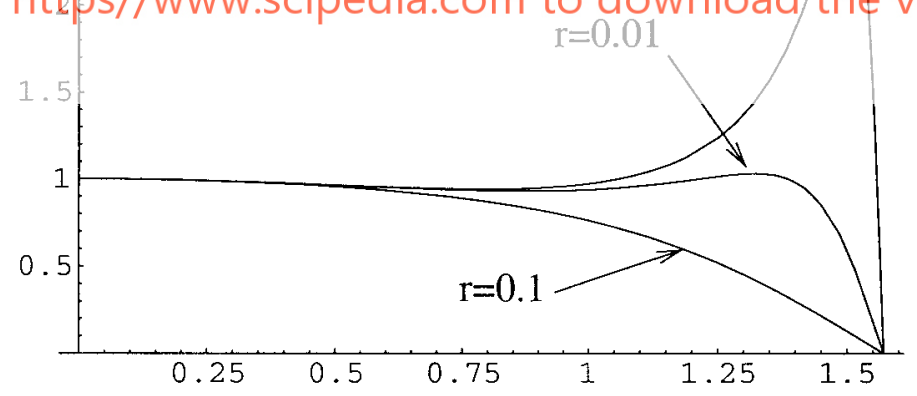

Figure 1. $S_{G}$ versus $\theta$ for the GLS method with $r=10^{-1}, 10^{-2}$ and $10^{-3}$. 


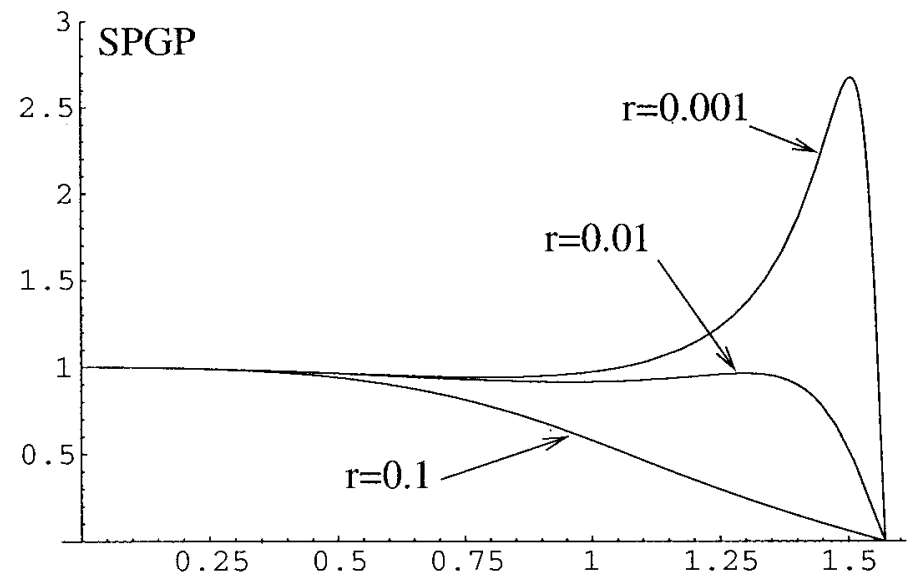

Figure 2. $S_{G}$ versus $\theta$ for the SPGP method with $r=10^{-1}, 10^{-2}$ and $10^{-3}$.

value of 1 , for $\theta$ as low as 0.75 . For $r=10^{-3}, S_{G}$ exhibits a peak near the mesh mode $(\theta=\pi / 2)$, proving that the spurious mode of the Galerkin formulation is not properly stabilized. From the observation that $r$

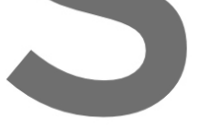
$\frac{\tau_{p}}{\rho}=\alpha=\frac{\tilde{\alpha} h^{2}}{\mu^{\prime}}$

it follows that a good choice for $\tau_{p} / \rho$ is Register for free at https//www.Scipedia.com to download the version without the watermar

$$
\frac{\tau_{p}}{\rho}=\frac{h^{2}}{100 \mu^{\prime}}
$$

for both methods, which is consistent with usual adopted formulae for GLS [2].

\section{Remark}

Another possibility, by inspection of Equation (35), is to put $\tilde{\gamma}+\tilde{\delta}-\tilde{\alpha}=\frac{1}{24}$. This sets the second-order term in $S_{G}$ to zero, leaving a zeroth-order term in $S_{V}$. Though no further investigation has yet been performed, the increase in accuracy for the most sensitive variable (the pressure) may prove useful. (Accuracy of order eight in $G$ is obtained choosing $\tilde{\gamma}=\tilde{\alpha}-$ $\tilde{\delta}+\frac{1}{24}, \tilde{\delta}=2 \tilde{\alpha}-\frac{1}{48}$ and $\left.\tilde{\alpha}=\frac{1}{32} ! ! !\right)$ 


\section{START-UP FLOW AROUND A CYLINDER: SPURIOUS PRESSURE WAVE}

To motivate the von Neumann's analysis of the next section, we briefly report here on a numerical test. The problem consists of the start-up flow around a circular cylinder at $R e=3000$, defined as $R e=\rho U_{\infty} D / \mu, U_{\infty}$ being the unperturbed flow velocity and $D$ the cylinder's diameter. The domain is $\Omega=(-5,15) \times(-5,5) \backslash C$, with $C$ being the cylinder of unit diameter centred at the origin. This problem has been studied both experimentally [23] and numerically (by the GSMAC method [24]). Starting from rest, $U_{\infty}$ is impulsively modified to $U_{\infty}=1$, with $D=1, \rho=3000$ and $\mu=1$. Attempts to simulate this flow starting from rest systematically failed. Success was attained using as initial condition the inviscid solution, as discussed by Gresho [25], i.e.

$$
u_{1}=1-\frac{x_{1}^{2}-x_{2}^{2}}{4 r^{4}}, \quad u_{2}=-\frac{x_{1} x_{2}}{2 r^{4}}, \quad p=\frac{\rho\left(x_{1}^{2}-x_{2}^{2}-\frac{1}{8}\right)}{2 r^{4}}
$$

where $r^{2}=x_{1}^{2}+x_{2}^{2}$. The flow is characterized by the formation and growth of two main symmetric vortices downstream of the cylinder, with some secondary vortices also appearing (see Figure 3). The simulation time is 3 units, with a time step of $\Delta t=0.001$. We do not intend to give detailed results here. The numerical parameters were $\tau_{u}$ according to Equation (12), $\tau_{p}=\tau_{u}, \beta=0$. The mesh consisted of $11518 P_{1}$ triangles, refined near the cylinder. The main fact is that the velocity field is accurately predicted. A snapshot of the velocity field at $t=3$ can be seen in Figure 4, which compares well with experimental data. In Figure 5, a quantitative comparison of the time evolution of the flow characteristic lengths $a, b$ and $L$ (see Figure 3) with the experiment is shown
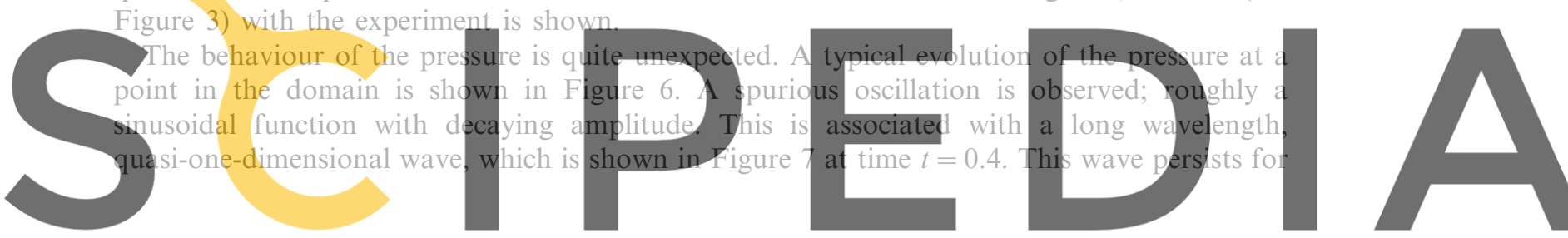

Register for free at https//www.scipedia.com to download the version without the watermar

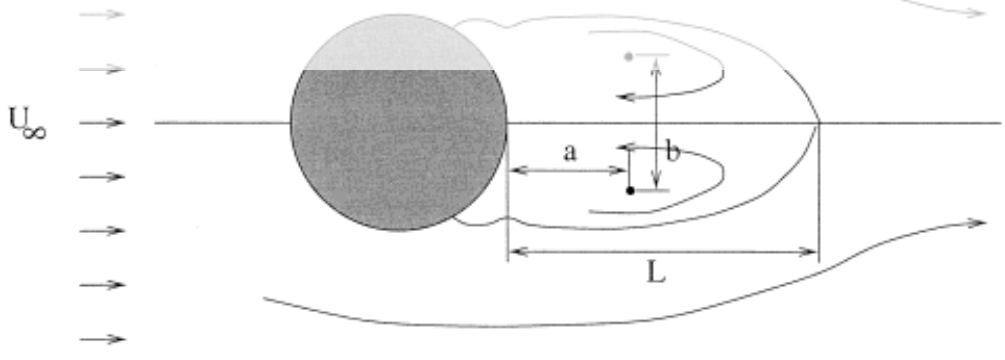

Figure 3. Problem geometry and definition of $a, b$ and $L$. 

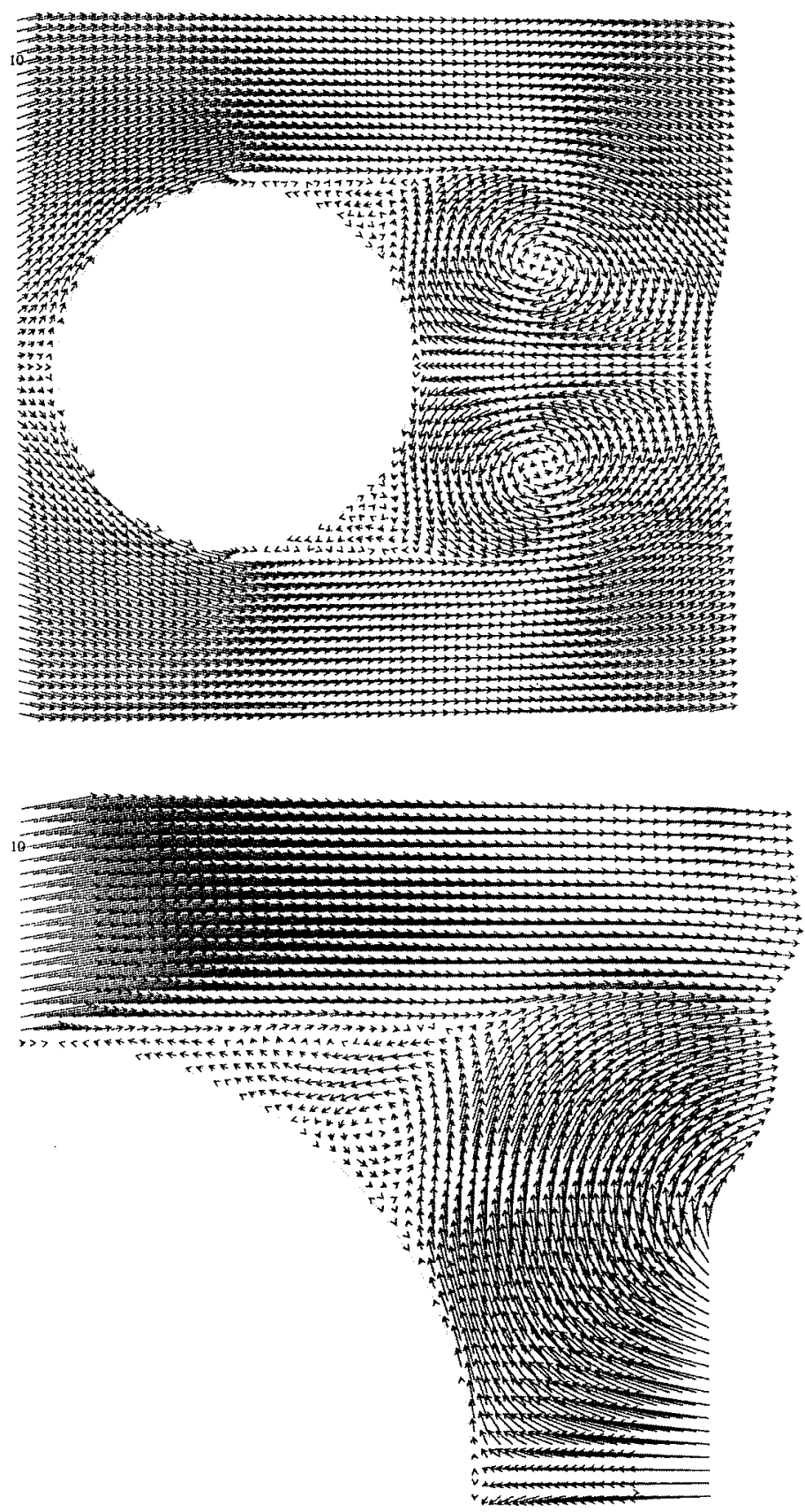

Figure 4. Velocity field at $t=3$ and detail showing secondary vortices. 


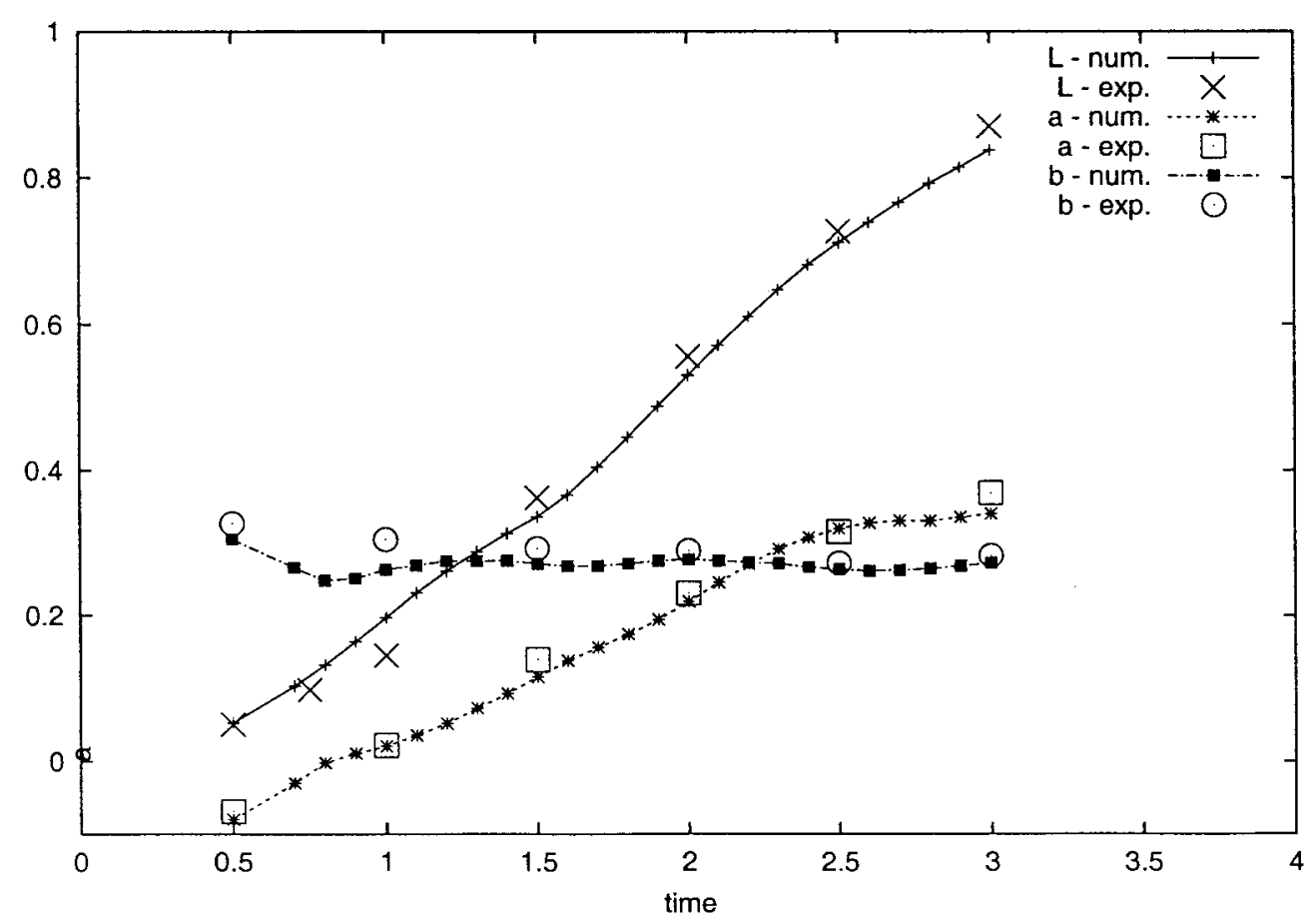

Figure 5. Comparison of numerically obtained time evolution of $L, a$ and $b$ with experimental data.

approximately 1 time unit and is superposed to the correct pressure field, though it does not affect the velocity field since its gradient is small. The decay time is empirically seen to be almost independent of $\Delta t$. This numerical artifact motivated the von Neumann analysis reported in the next section. It should be kept in mind, however, that for less severe transients no spurious wave appears. The periodic vortex shedding at $R e=100,200$ and 400, for example, can be accurately simulated.

\section{VON NEUMANN'S ANALYSIS OF THE TRANSIENT CASE}

\subsection{Stability analysis}

The stability of the SPGP method has been addressed by Codina and Blasco [15] for the Navier-Stokes problem using variational methods.

Let us review the main stability result of this reference here. For simplicity, consider the case $g=0$ in Equation (5) and $\Gamma_{\mathrm{N}}=\varnothing$. Omitting as before, the SUPG terms $\left(\tau_{u}=0\right)$ and assuming $\tau_{p}$ to be $O\left(h^{2}\right)$, it can be shown that the discrete solution of problem (9)-(11) satisfies 


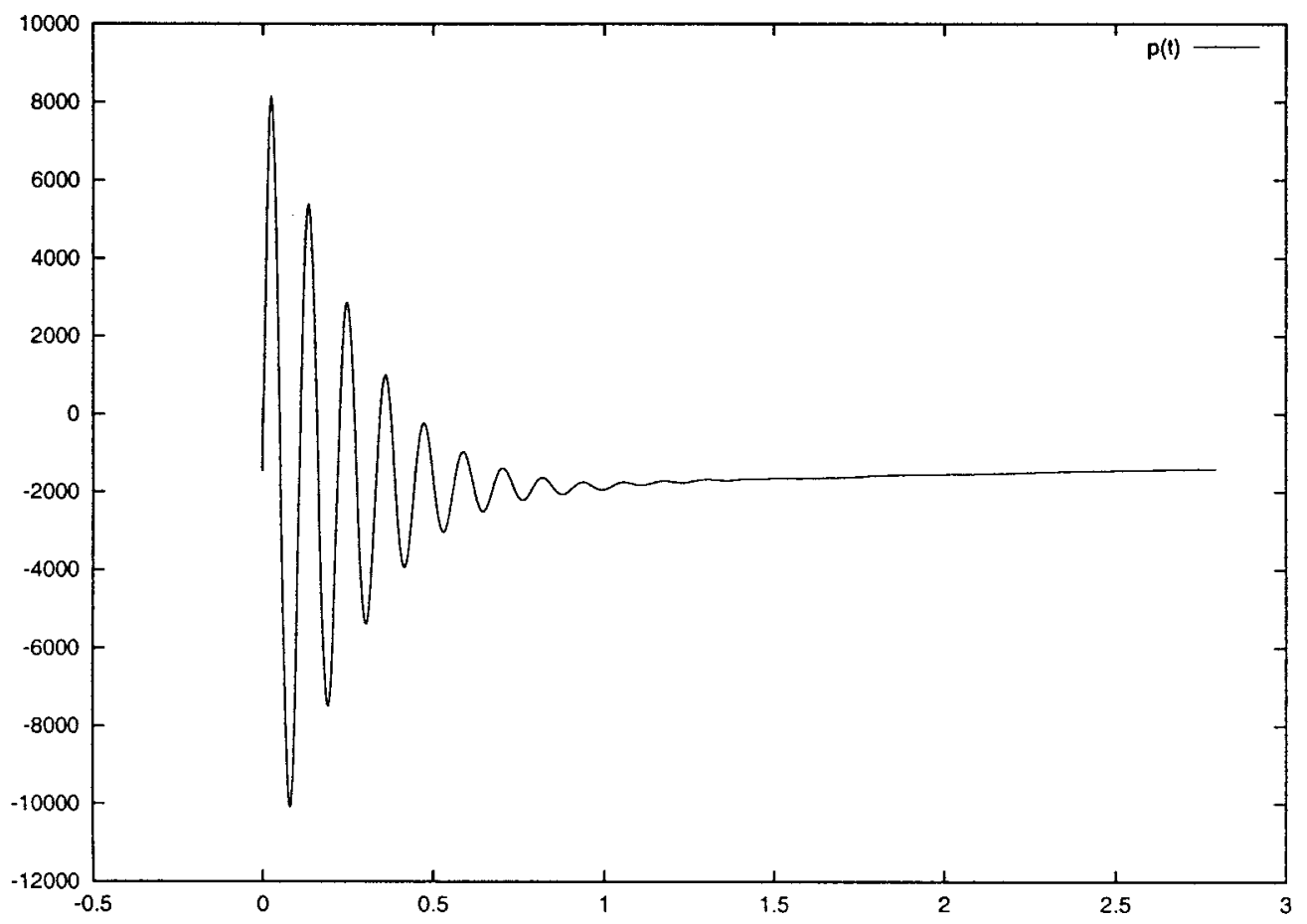

Figure 6. Typical evolution of pressure at a point.

$$
\left\|u_{h}^{n+1}\right\|^{2}+v \Delta t \sum_{k=0}^{n}\left\|\nabla u_{h}^{k}\right\|^{2}+\Delta t h \sum_{k=0}^{n}\left\|\nabla p_{h}^{k}\right\| \leq C \Delta t \sum_{k=0}^{n}\left\|f^{k}\right\|^{2}
$$

where $v=\mu / \rho$ is the kinematic viscosity, $\|\cdot\|$ is the $L^{2}$-norm on the domain $\Omega$ and $C$ is a constant that depends on $\Omega$ and $v$ but not on the mesh size $h$ or on the time step size $\Delta t$. According to Equation (41), $\left\{\left\|u_{h}^{k}\right\|\right\}$ is an $\ell^{\infty}$ sequence, $\left\{\left\|\nabla u_{h}^{k}\right\|\right\}$ is an $\ell^{2}$ sequence and $\left\{h\left\|\nabla p_{h}^{k}\right\|\right\}$ is an $\ell^{1}$ sequence whenever $\left\{\left\|f^{k}\right\|\right\}$ is $\ell^{2}$ (the stability for the pressure can be improved to $\ell^{2}$ for two-dimensional flows and for the Stokes problem; see Reference [15]). Even though Equation (41) provides all the information that one can expect from a global stability estimate, it is silent in what concerns local temporal behaviour and possible limiting cases. To gain insight into these points, we address here the stability analysis of the SPGP method for the one-dimensional model problem (24) and (25) using von Neumann's technique. The advantage of this simplified setting (linear, one-dimensioanl, domain $=(-\infty,+\infty)$ ) is that it is possible to scrutinize the algorithm's temporal behaviour; but of course the assumption that the Navier-Stokes problem will behave alike is not rigorous. However, in the next paragraphs we show some evidence in this direction.

Consider the stencil (26) and (27). It corresponds to the SPGP method as analysed in Reference [15]. The momentum equation is discretized using Galerkin's method, while the incompressibility constraint has been replaced by (from Equations (16) and (17)) 


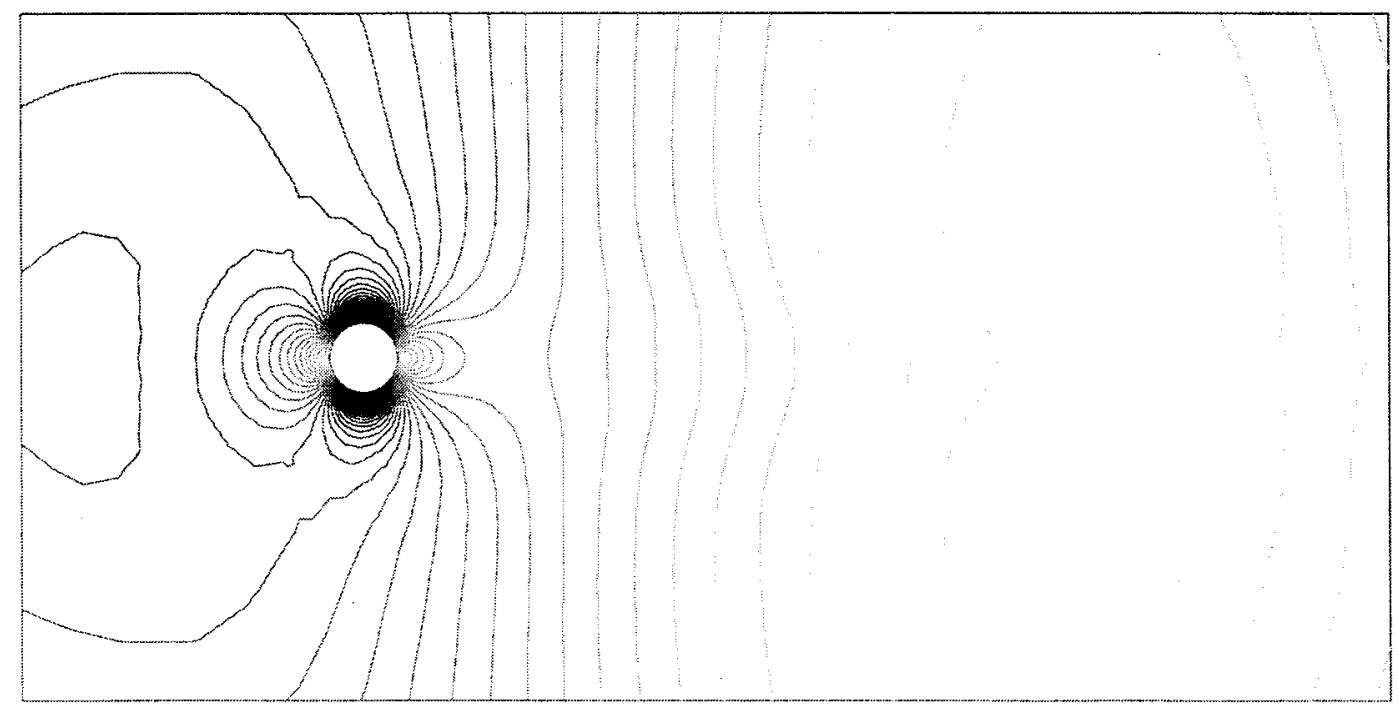

pmin $=-5583.93, \operatorname{pmax}=260.14$

Figure 7. Pressure field at time $t=0.4$. Notice the spurious pressure wave, with wavelength of the order of the domain size.

$$
\mathbf{B}^{T} \underline{U}^{n}+\frac{1}{\rho} \mathbf{L} \underline{P}^{n}=\frac{1}{\rho} \mathbf{D}^{T} \mathbf{M}^{-1} \mathbf{C} \underline{P}^{n-1+\beta}
$$

We discuss first the effects of $\beta$ and of SUPG treatment of the momentum equation $\left(\tau_{u}>0\right)$.

After the substitution

$$
\begin{aligned}
U_{i}^{n} & \rightarrow A r^{n} \mathrm{e}^{-\mathrm{I} i k h} \\
P_{i}^{n} & \rightarrow B r^{n} \mathrm{e}^{-\mathrm{I} i k h}
\end{aligned}
$$

( $r$ is now the amplification factor) we obtain a homogeneous linear system of two algebraic equations for the two unknowns $A$ and $B$. To avoid trivial solutions, the determinant of the system must vanish. This condition provides a relation between $r$ and the six free parameters $\Delta t, h, c, \rho, \mu, k$, from which the two eigenvalues (denoted $r_{1}$ and $r_{2}$ ) were obtained by symbolic manipulation using Mathematica [22]. The number of parameters can be conveniently reduced to three by introducing non-dimensional quantities, such as the mesh Reynolds number, $R e_{h}=\rho c h / \mu$, the Courant-Friedrich-Lewy (CFL) number, $C=c \Delta t / h$ and the nondimensional wavenumber $\kappa=k h$.

By inspection of Mathematica's plots, and as predicted in Reference [15], the SPGP method is stable $(|r|<1)$ both for $\beta=0$ and $\beta=1$. Graphs of $|r|:=\max \left\{\left|r_{1}\right|,\left|r_{2}\right|\right\}$ for the case $\beta=0$ are shown in Figure 8(a) and (b). Part (a) corresponds to $R e_{h}=0.01$, and three values of $C$ are 
considered: $0.05,0.5$ and 5. The same is done in part (b) for $R e_{h}=100$. Owing to the incompressibility constraint (in one-dimensional domains), both eigenvalues $r_{1}$ and $r_{2}$ are zero in the exact problem for any $k>0$. This means that waves of finite wavelength are instantaneously damped and only uniform (rigid) motions are allowed. However, $|r|$ is not zero in the discrete problem as shown in the graphs. In general, the eigenvalues are complex, so that the uniform motion is reached after a spurious oscillatory transient in which decaying waves are observed. For low $R e_{h}$ and waves with $k h \sim 2$, the amplitude is multiplied by $\sim 0.1$ at each time step. Long waves, $k h \sim 0$, become more persistent as the CFL decreases. This spurious behaviour aggravates in the high- $R e_{h}$ regime.

Both long and short waves become very persistent $(|r| \sim 1)$ for small CFL values. In Figure 9 the typical decay time $\tau$ of long waves relative to $\Delta t$ is shown as a function of CFL (for such long waves, $|r|$ is independent of $R e_{h}, \tau$ is defined as the time elapsed before the amplitude of the wave reduces to $1 \%$ of its initial value). Notice that $\tau / \Delta t \rightarrow \infty$ as CFL $\rightarrow 0$. This
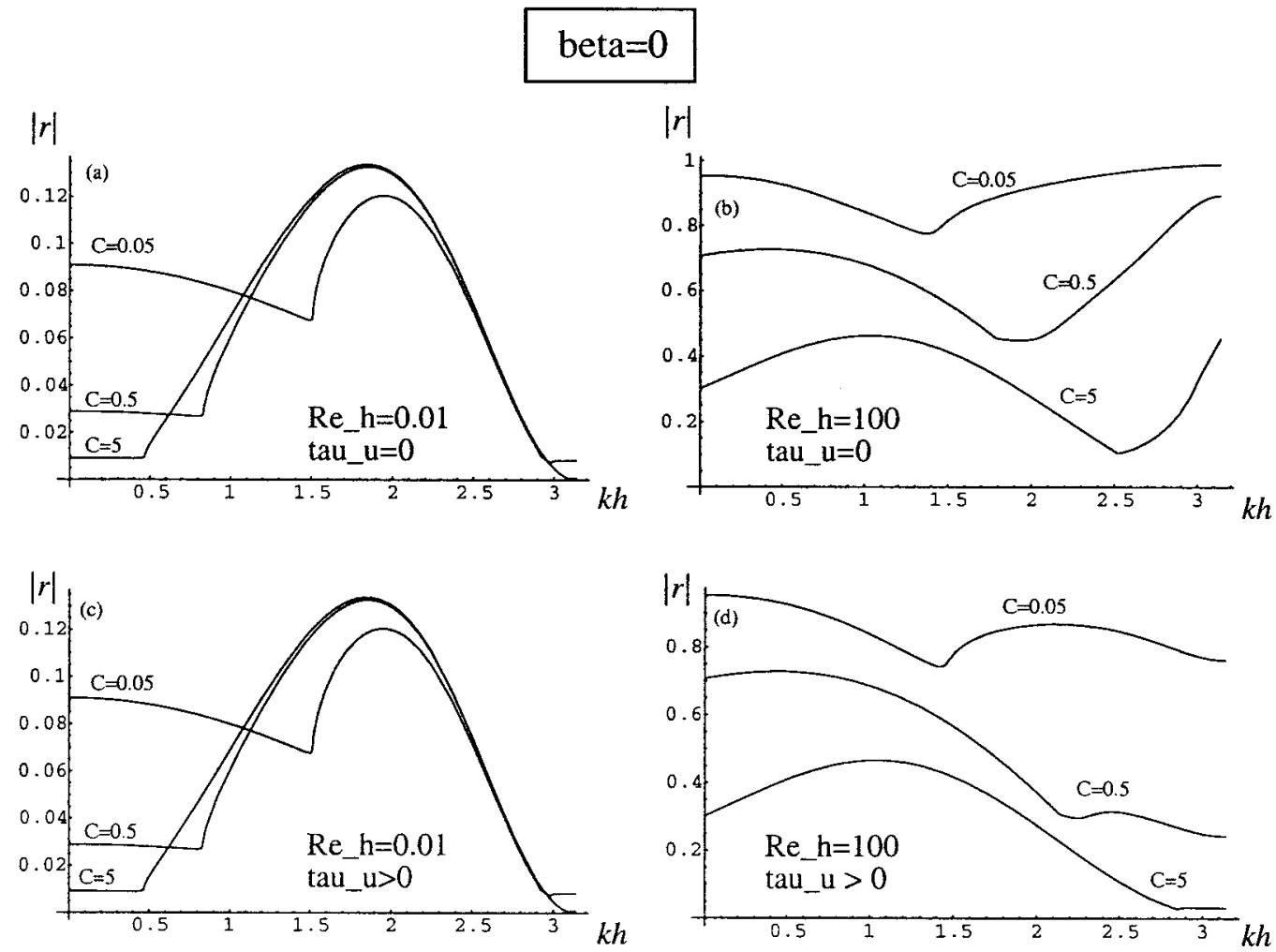

Figure 8. Amplification factor of the SPGP method for the case $\beta=0$. Two values of $R e_{h}$ are considered (0.01 and 100), and three values of the CFL number $(C=0.05,0.5,5)$. Treatment of the momentum equation: (a) and (b) Galerkin; (c) and (d) SUPG. 
phenomenon constitutes a remarkable spurious temporal behaviour of the SPGP method with explicit treatment of the pressure gradient projection $(\beta=0)$. No such behaviour is found, as shown later on, if the pressure gradient projection is dealt with implicitly $(\beta=1)$ or if GLS or Chorin's stabilization techniques are used, because in these cases $|r| \rightarrow 0$ when $k h \rightarrow 0$.

Figure 8(c) and (d) shows the effect of adding SUPG stabilization to the momentum equation. The low- $R e_{h}$ regime remains the same as expected. For high $R e_{h}$, the damping of short waves $(k h \sim \pi)$ is increased, but no improvement is brought to longwave damping. It has to be remarked that convection must be taken into account in the design of $\tau_{p}$. In fact, if the expression $\tau_{p}=\rho h^{2} / 12 \mu$ used in Reference [15] (based on the viscous-dominated case) is adopted, the situation gets worse.

One concludes that, though the SPGP method with $\beta=0$ is indeed stable (with and without SUPG in the momentum equation), spurious transients appear that may pollute the results during many time steps, especially in those regions of the domain where the CFL is smallest (because of larger mesh size or smaller velocity). Notice that this already explains the spurious behaviour of Section 4. In fact, far from the cylinder, the mesh used had $h \sim 0.3$ leading to

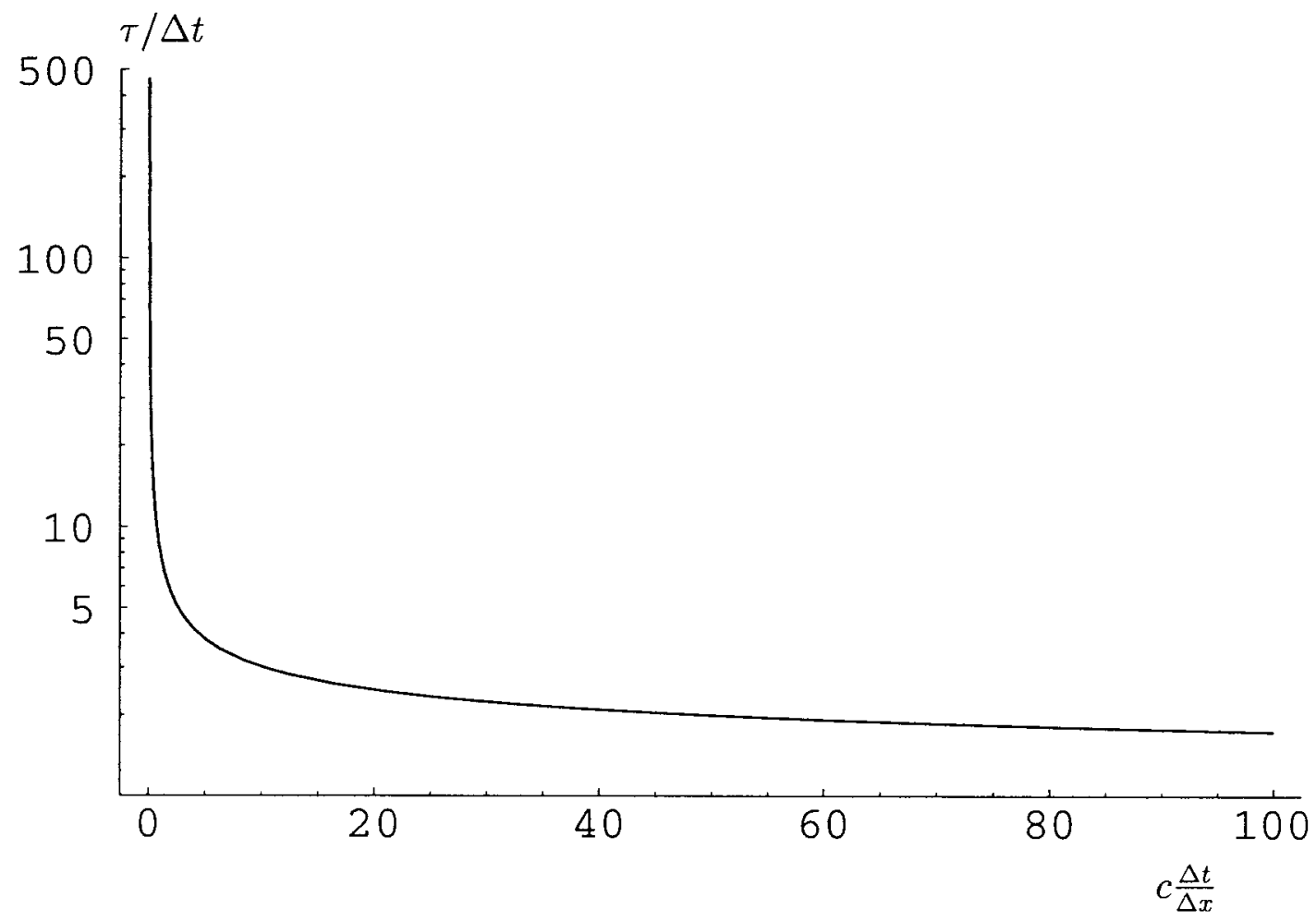

Figure 9. Long waves $(k=0) . \tau / \Delta t$ versus Courant's number $(\tau / \Delta t$ is independent of the Reynolds number). SPGP method, $\beta=0$, no upwinding. 
$R e_{h} \sim 900$ and $C \sim 0.003$, values that fall well within the expected range of longwave persistance.

As said above, let us now show that this phenomenon is linked to the choice $\beta=0$. In Figure 10 we show similar plots for $\beta=1$. SUPG stabilization has been applied to the momentum equation to damp the short waves (velocity wiggles). In the low- $R e_{h}$ regime all wavelengths are strongly damped $(|r|<0.01)$. For high $R e_{h}$ and low CFL some persistence of waves in the range $0.1<k h<\pi$ is predicted, but $|r|$ is small enough $(|r| \sim 0.8$ or lower $)$ to damp perturbations after a few $(\sim 30)$ time steps.

A cure for long spurious transients is thus to take $\beta=1$ in Equation (42). This is, however, not practical as the computational cost becomes prohibitive. An alternative may come from extrapolation. We have considered two possibilities

\section{EXTRAP1}

$$
\mathbf{D}^{T} \mathbf{M}^{-1} \mathbf{C} \underline{P}^{n} \sim \mathbf{D}^{T} \mathbf{M}^{-1} \mathbf{C}\left(2 \underline{P}^{n-1}-\underline{P}^{n-2}\right)
$$

\section{EXTRAP2}

$$
\mathbf{D}^{T} \mathbf{M}^{-1} \mathbf{C} \underline{P}^{n} \sim 2 \mathbf{D}^{T} \mathbf{M}^{-1} \mathbf{C} \underline{P}^{n-1}-\mathbf{L} \underline{P}^{n-2}
$$

Unfortunately, both are unstable. Plots of $|r|$ are shown in Figure 11. Irrespective of $R e_{h}$ and $C$ there exist waves with $|r|>1$. Notice that, as the scheme now involves three time levels, $|r|$ is the maximum of the moduli of four eigenvalues. In agreement with these predictions, when these extrapolation schemes were programmed into the Navier-Stokes code, exponential instability was found.

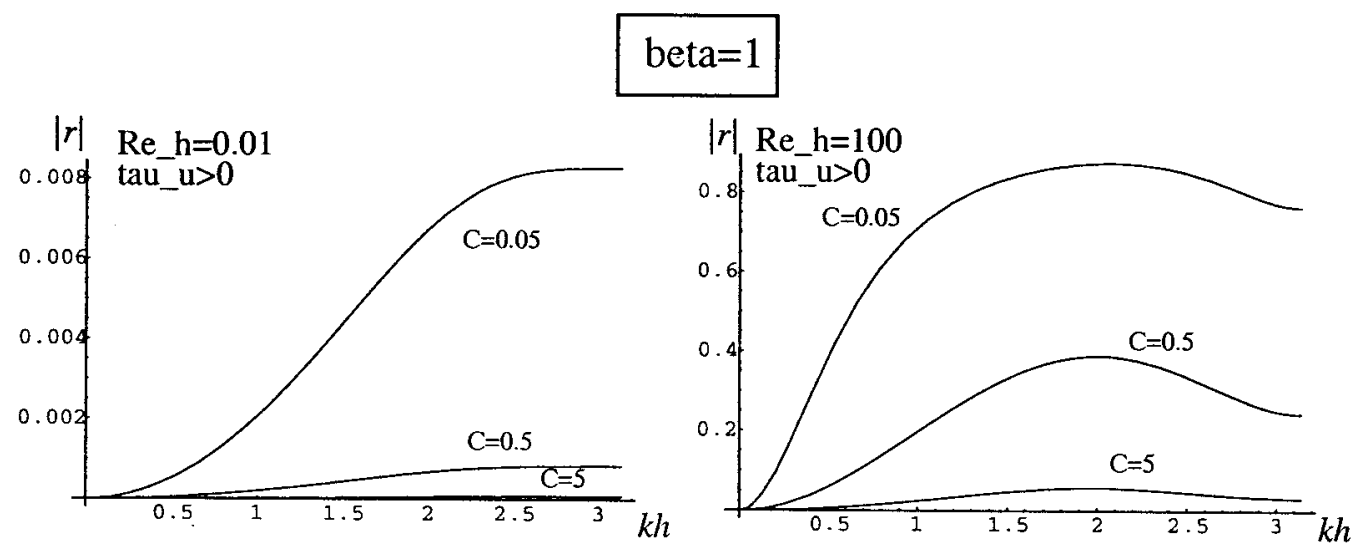

Figure 10. Amplification factor of the SPGP method for the case $\beta=1$. Two values of $R e_{h}$ are considered (0.01 and 100), and three values of the CFL number $(C=0.05,0.5,5)$. Treatment of the momentum equation: SUPG. 


\subsection{Comparison with the GLS and Chorin's methods}

It is interesting to compare the results above with those corresponding to the GLS and Chorin's methods (see stencils in Appendix A). The amplification factors $|r|$ for these methods are plotted as functions of $k h$ for the high-Re and low-Re regimes in Figure 12. Both have $|r| \rightarrow 0$ as $k h \rightarrow 0$. Both are stable and lead to some (in fact small) persistence of waves only if $R e_{h}$ is large and $C$ small, as does the SPGP method, with $\beta=1$. Least prone to this phenomenon is Chorin's method (the short waves with $k h \sim \pi$ can be damped by introducing upwinding in the momentum equation, which was not done). The predictions are, however, comparable for the three methods.

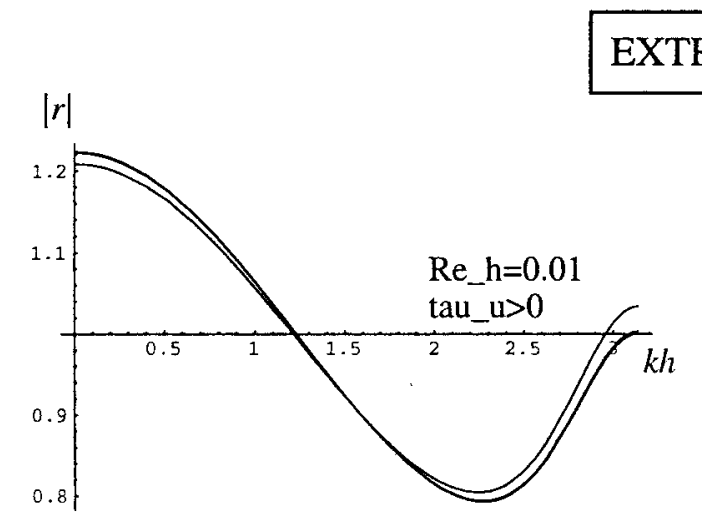

EXTRAP1

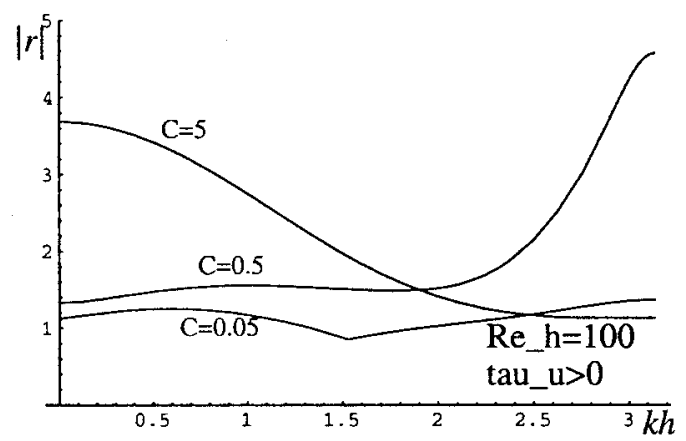

EXTRAP2
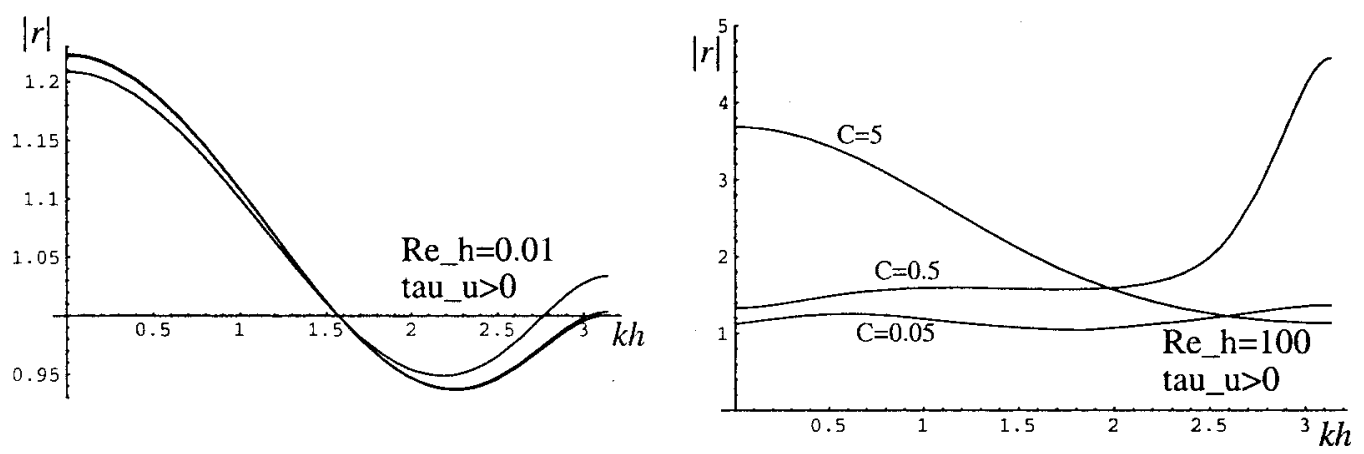

Figure 11. Amplification factor of the SPGP method for extrapolations EXTRAP1 and EXTRAP2. Two values of $R e_{h}$ are considered (0.01 and 100), and three values of the CFL number $(C=0.05,0.5,5)$.

Treatment of the momentum equation: SUPG. 


\subsection{One more variant: stabilization by residual projection}

The SPGP method, in the case of Stokes flow, can be regarded as a modification of the GLS method as follows. Let the residual of $\left(u_{h}, p_{h}\right) \in V_{h} \times Q_{h}$ as regards the momentum equation be defined by

$$
\mathscr{R}\left(u_{h}, p_{h}\right)=-2 \operatorname{div}\left(\mu D u_{h}\right)+\nabla p_{h}-f=\mathscr{L}\left(u_{h}, p_{h}\right)-f
$$

then the GLS equations are

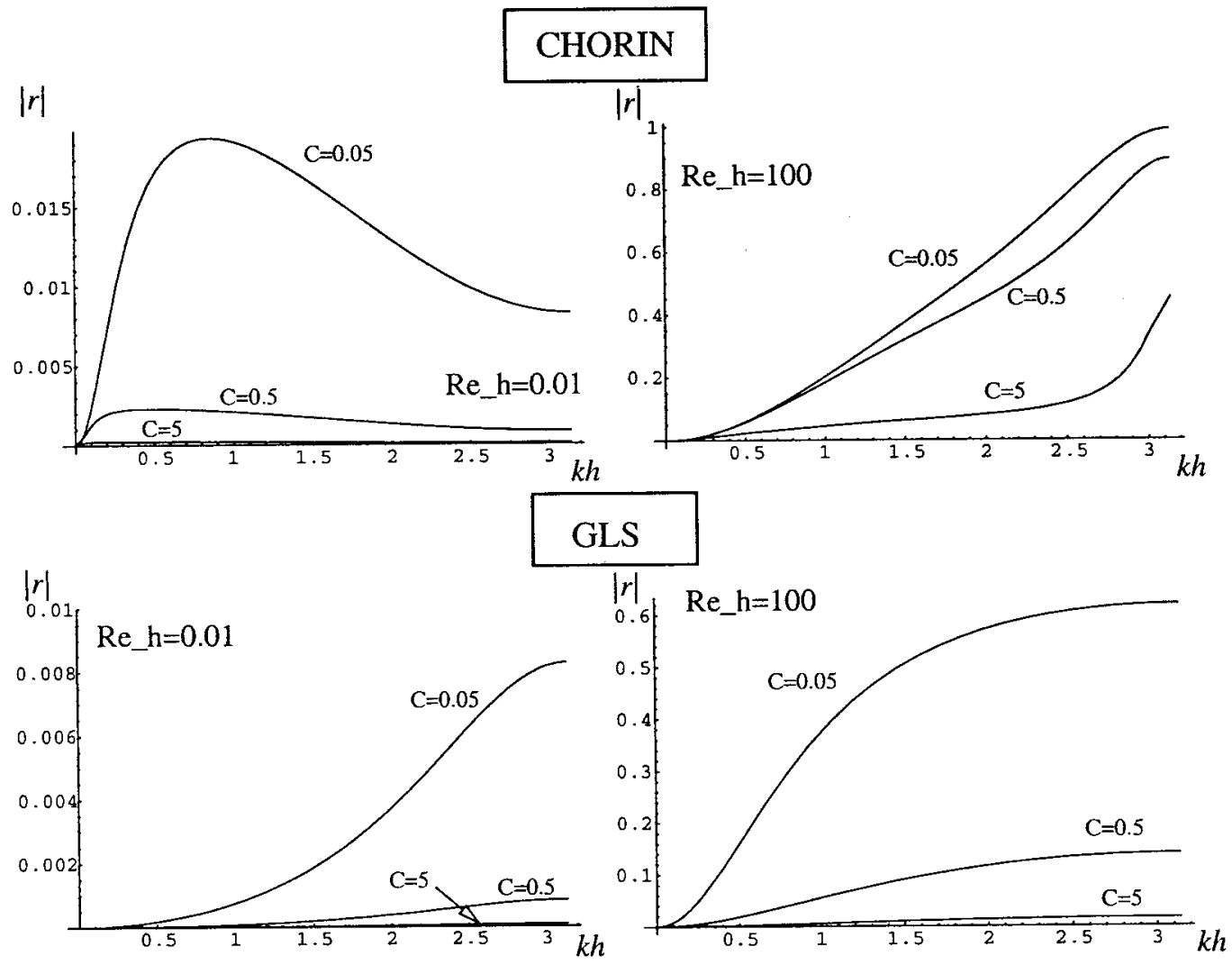

Figure 12. Amplification factor of Chorin's and the GLS methods. Two values of $R e_{h}$ are considered (0.01 and 100), and three values of the CFL number $(C=0.05,0.5,5)$. 


$$
\begin{aligned}
& a\left(u_{h}, v_{h}\right)-\left(p_{h}, \operatorname{div} v_{h}\right)-\left(f, v_{h}\right)-\int_{\Gamma_{\mathrm{N}}} \mathscr{F} \cdot v_{h} \mathrm{~d} \Gamma+\left(q_{h}, \operatorname{div} u_{h}\right) \\
& +\sum_{K \in \mathscr{T}_{h}}\left(\mathscr{R}\left(u_{h}, p_{h}\right), \frac{\tau_{u}}{\rho} \mathscr{L}\left(v_{h}, q_{h}\right)\right)_{K}=0
\end{aligned}
$$

On the other hand, for linear elements, piecewise linear $f$ and Stokes flow, the SPGP method can be written as

$$
\begin{aligned}
& a\left(u_{h}, v_{h}\right)-\left(p_{h}, \operatorname{div} v_{h}\right)-\left(f, v_{h}\right)-\int_{\Gamma_{\mathrm{N}}} \mathscr{F} \cdot v_{h} \mathrm{~d} \Gamma+\left(q_{h}, \operatorname{div} u_{h}\right) \\
& +\sum_{K \in \mathscr{T}_{h}}\left(\mathscr{R}\left(u_{h}, p_{h}\right)-\Pi_{h} \mathscr{R}\left(u_{h}, p_{h}\right), \frac{\tau}{\rho} \mathscr{L}\left(v_{h}, q_{h}\right)\right)_{K}=0
\end{aligned}
$$

It is evident that the only difference with the GLS equations is that the projection of the residual, $\Pi_{h} \mathscr{R}$, onto $V_{h}$ has been subtracted from the residual itself within the stabilization term. Consistency has not been lost, since for the exact solution the residual vanishes. With this interpretation one could proceed analogously for the transient Navier-Stokes problem, arriving at a variant of the SPGP method. In this case, the residual is

$$
\mathscr{R}\left(u_{h}, p_{h}\right)=\rho \frac{\partial u_{h}}{\partial t}+\rho\left(u_{h} \cdot \nabla\right) u_{h}-2 \operatorname{div}\left(\mu D u_{h}\right)+\nabla p_{h}-f
$$

For non-zero $R e$, the residual of the incompressibility constraint also enters the GLS formulation [1], times a mesh-dependent constant

$$
\delta_{K}=\rho\left|u_{h}\right|^{2} \tau_{u}
$$

and thus, assuming linear elements, the stabilization terms are

$$
\begin{aligned}
& \sum_{K \in \mathscr{T}_{h}}\left(\left(\rho\left(u_{h} \cdot \nabla\right) u_{h}+\nabla p_{h}\right)-\Pi_{h}\left(\rho\left(u_{h} \cdot \nabla\right) u_{h}+\nabla p_{h}\right), \frac{\tau_{u}}{\rho}\left(\rho\left(u_{h} \cdot \nabla\right) v_{h}+\nabla q_{h}\right)\right)_{K} \\
& +\sum_{K \in \mathscr{T}_{h}} \delta_{K}\left(\operatorname{div} u_{h}-\Pi_{h} \operatorname{div} u_{h}, \operatorname{div} v_{h}\right)_{K}
\end{aligned}
$$

An almost equivalent formulation is proposed in Codina [17], where it is shown that it is in fact a SGS method [18] with a particular choice for the space of sub-scales.

von Neumann's analysis of this method yields results that resemble those of the SPGP method. In this case, the possibility also appears of dealing with the residual projection either implicitly ( $\beta=1$ in the SPGP method) or explicitly $(\beta=0)$, and again the only reasonable choice (from the computational point of view) is the latter. The results are shown in Figure 13. 


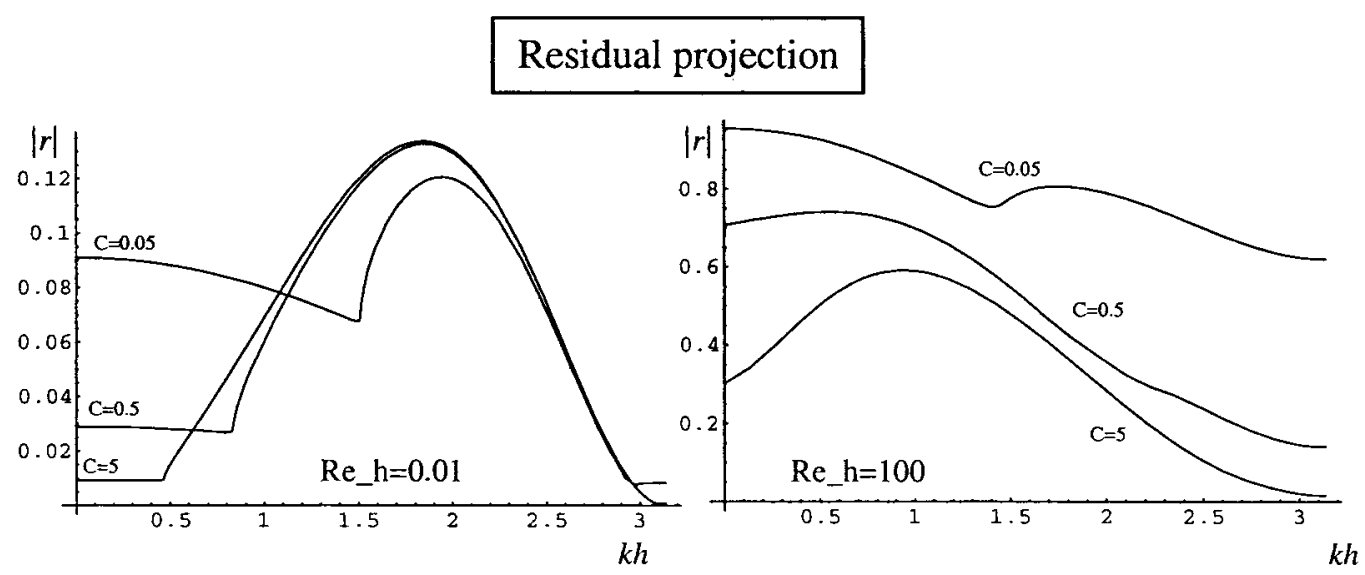

Figure 13. Amplification factor of full-residual projection variant of the SPGP method. Two values of $R e_{h}$ are considered $(0.01$ and 100$)$, and three values of the CFL number $(C=0.05,0.5,5)$.

\section{NUMERICAL TESTS}

The predictions of the previous section are based on the linearized version of the model problem (24) and (25), and disregarding boundary effects. It is important to check that the conclusions are not overruled as soon as these simplifying hypotheses are dropped.

\subsection{Comparison for a one-dimensional model problem}

Considering first the full (non-linear) one-dimensional model problem (24) and (25), to be solved for $t>0$ in $\Omega=(0,1)$ with $f=0$ and initial and boundary conditions

$$
u(x, t=0)=0, \quad u(0, t>0)=1, \quad p(1, t>0)=0
$$

the exact solution is

$$
\begin{aligned}
& u(x, t)=1 \\
& p(x, t)=0
\end{aligned}
$$

A series of numerical experiments on this problem were performed using the SPGP method. Linear one-dimensional finite elements were used. Experiences were made with $\rho=1, \mu=$ $1 / 45000$, for different values of $h$ and $\Delta t$. The computed pressure is seen to oscillate around zero with an amplitude that decays to zero. This is a spurious pressure transient that is activated by the sudden imposition of $u=1$ on the left boundary. The period of the oscillations, and the decay behaviour, are not predictable a priori due to the non-linearity of the problem. Our aim here is to compare them with the predictions from von Neumann's analysis (which rigorously apply just to the linearized model with no boundary). 
In Figure 14 we present results for some selected cases. Plotted is the value of the pressure at $x=0$. The solid lines correspond to the numerical experiments and the dashed ones to von Neumann's analysis predictions. To be precise, the dashed lines correspond to (the real part of) the functions

$$
u(x=0, n \Delta t)=A r^{n}, \quad p(x=0, n \Delta t)=B r^{n}
$$

where $r$ is the (complex) amplification factor of the method. As von Neumann's analysis does not predict the initial magnitudes and phases of $u$ and $p$ (i.e. $A$ and $B$ ), these have been adjusted so that they coincide with the numerical result at the first positive peak. The value of $r$ was obtained with Mathematica for the values of $h, \Delta t, \mu$ and $\rho$ used in each simulation. The wavenumber $k$ was assigned the value zero, since long waves are the most persistent ones for the cases considered, and $c$ was set to 1 .

The values of $\Delta t$ and $h$ are shown in the inserts of each graph. Except for a short initial transient (probably dominated by non-linear effects), the computed pressure exhibits practically the same temporal behaviour as the one predicted by von Neumann's analysis. Both the oscillation period and the decay time are in good agreement. This supports the use of the results of the previous section to draw conclusions about the temporal behaviour of the SPGP method in a broader class of cases than that to which the von Neumann's analysis rigorously applies.

\subsection{Comparison with two-dimensional start-up flow results}

Finally, let us analyse the more interesting situation of the two-dimensional start-up flow around a cylinder solved by finite elements, as described in Section 4. Parameters for this case were taken from the two-dimensional problem: $h=0.3, C=c \Delta t / h=3.33 \times 10^{-3}, R e_{h}=900$. As in this case we have again a high $R e_{h}$ and a low $C$, von Neumann's analysis predicts that the most persistent wave would correspond to $k=0$. In Figure 15 the full line represents the computed pressure value behind the cylinder. The dashed line is the real part of $A r^{n}$, with $n=t / \Delta t$ and $r$ being the amplification factor corresponding to the parameters listed above. This time, the continuous and dashed curves were put into coincidence (by adjusting $A$ ) at the second positive peak because during the first period of the simulation a second mode can be observed, superimposed to the most persistent, longwave one.

It can be appreciated that both extinction time and period of oscillation compare well with (though being slightly larger than) those of the finite element calculation. In spite of the differences between both situations (two-dimensional versus one-dimensional, non-linearity versus linearity, irregular versus regular meshes, different geometries and boundary conditions, etc.), the qualitative and quantitative coincidences are quite acceptable. The simple model correctly captures the basic features of this spurious wave propagation phenomenon.

It is worth pointing out that this kind of ghost pressure waves, which during a simulation can be activated by different unexpected causes, are quite sensitive to iteration stopping tolerances in either the non-linear or the linear solver. The simulation reported here used very stringent convergence criteria (relative tolerances of $10^{-7}$ ). If these are relaxed, the numerical system becomes 'softer' and the spurious waves tend to persist even longer. 


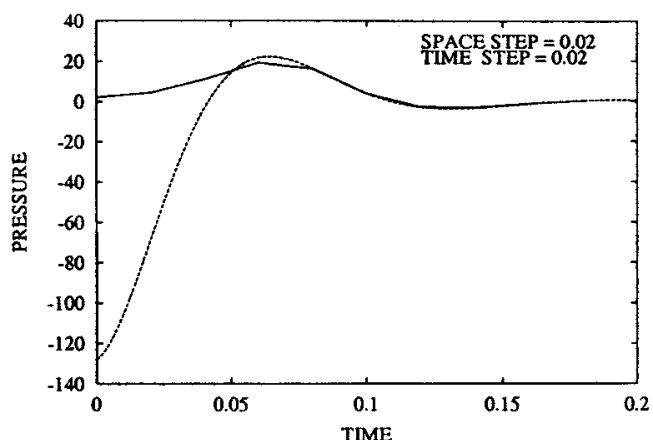

(a)

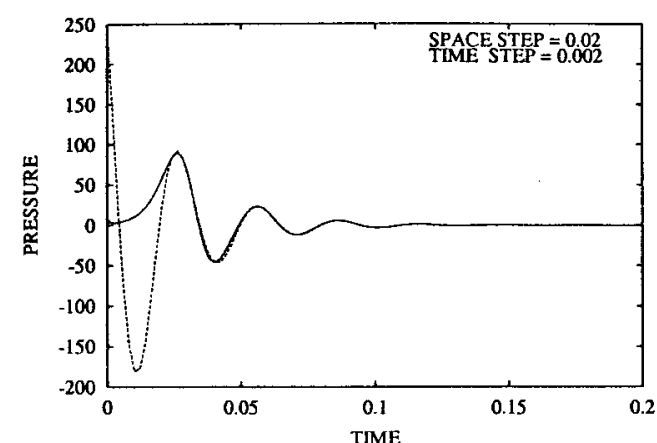

(b)

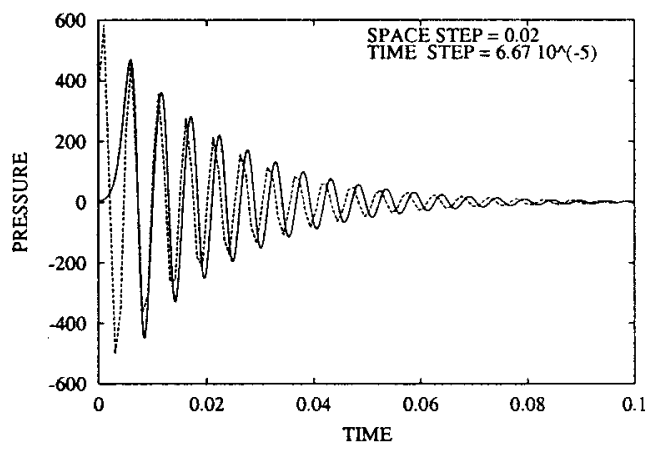

(c)

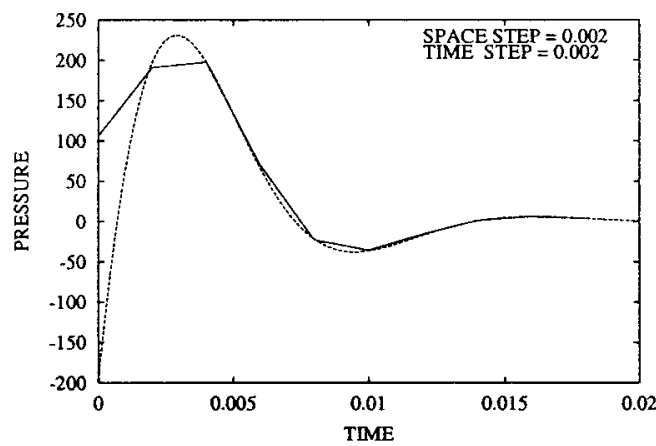

(d)

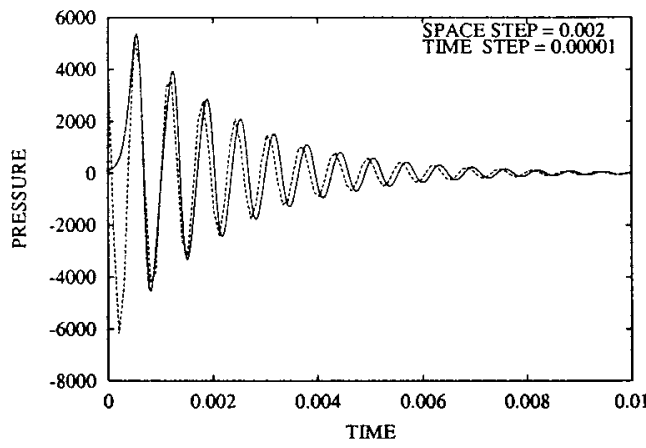

(e)

Figure 14. Pressure at $x=0$ versus time. Numerical experiments with the explicit SPGP method in the unit one-dimensional interval $(\rho=c=1, \mu=45000)$. Solid line: numerical experiment; dashed line: theoretical prediction. Both curves have been made to coincide near the first positive peak. 


\section{CONCLUDING REMARKS}

A detailed Fourier analysis and several numerical tests have been reported on the recent SPGP method. These come to complement and extend previous theoretical results by Codina and Blasco [15]. Unconditional stability is predicted, irrespective of the choice of $\beta$. SUPG treatment of the momentum equation has no deleterious effect on stability and damps, as expected, velocity wiggles. In fact, a better choice of the stabilization parameter $\tau_{p}$ is the SUPG intrinsic time $\tau_{u}$ as given by expression (12). An alternative way of incorporating convection in the design of the intrinsic time can be found in Codina [20].

Numerical tests on start-up flow around a cylinder at $R e=3000$ show that the velocity field is accurately predicted. However, a spurious pressure transient appears, which pollutes the pressure field during about half of the simulated time. This phenomenon, not previously reported, should be considered when using the SPGP method for, e.g. drag calculations or fluid-structure interaction, where accuracy in the pressure field is needed. Though not discussed here, less demanding calculations such as vortex shedding at lower $\operatorname{Re}(\leq 400)$ have not shown this problem.

Fourier analysis clearly identifies these spurious transients as coming from explicit treatment of the pressure gradient projection $(\beta=0)$. It is predicted to be most critical when $R e_{h}$ is large and CFL small. This situation is typical of regions within the computational domain where the mesh is coarse or the velocity small (stagnant regions). No cure was found by means of extrapolation, since the scheme becomes unstable.

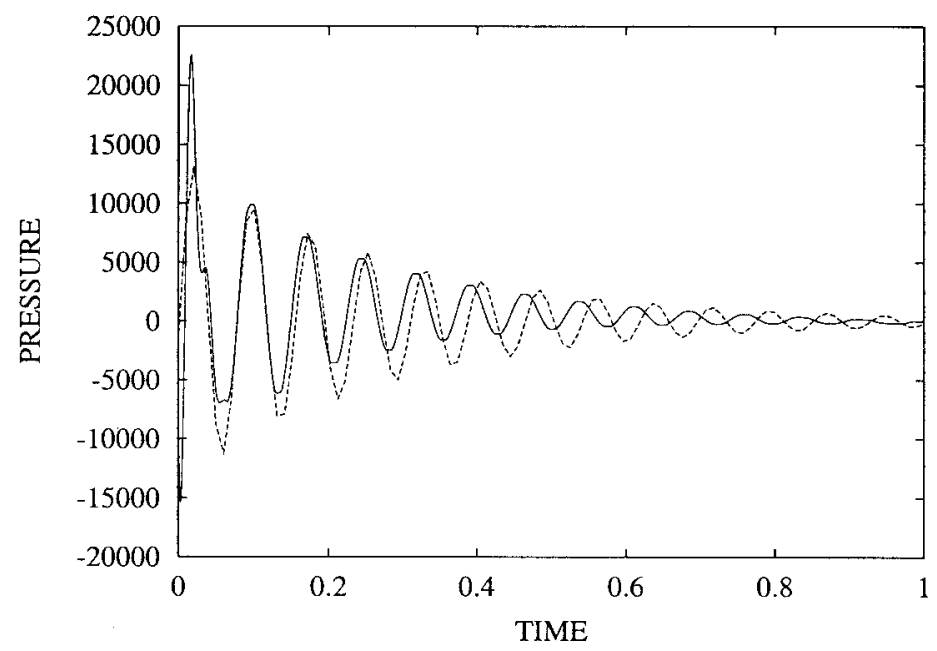

Figure 15. Start-up flow around a cylinder. Pressure behind the cylinder versus time. A comparison with theoretical one-dimensional values (dashed line). Both curves have been made to coincide at the second positive peak. 
Finally, it should be remarked that all predictions coming from Fourier analysis made above do not account for finite domain size and boundary conditions. Notice that trigonometric functions are not eigenfunctions of the exact problem in bounded domains unless periodicity is assumed. Heuristically, our approach has been to draw conclusions from the infinite-domain case and consider them applicable to more realistic situations. The numerical tests reported in the previous sections (especially those in Figure 14) support our approach, as remarkable coincidences between the predictions from von Neumann's analysis and actual computations have been obtained.

\section{ACKNOWLEDGMENTS}

This work was partially supported by Agencia Nacional de Promoción Científica y Tecnológica through grants PICT97 No. 12-00000-00982 and PMT-PICT 0392, BID 802/OC.

\section{APPENDIX A}

\section{A.1. Stencil of GLS method}

$$
\begin{aligned}
& \frac{\rho}{6 \Delta t}\left(U_{i-1}^{n}+4 U_{i}^{n}+U_{i+1}^{n}-U_{i-1}^{n-1}-4 U_{i}^{n-1}-U_{i+1}^{n-1}\right)+\rho c \frac{U_{i+1}^{n}-U_{i-1}^{n}}{2 h} \\
& -\left(2 \mu+\tau_{u} \rho c^{2}\right) \frac{U_{i+1}^{n}-2 U_{i}^{n}+U_{i-1}^{n}}{h^{2}}+\frac{P_{i+1}^{n}-P_{i-1}^{n}}{2 h}-\frac{\rho \tau_{u} c}{\Delta t} \frac{U_{i+1}^{n}-U_{i+1}^{n-1}-U_{i-1}^{n}+U_{i-1}^{n-1}}{2 h} \\
& -\tau_{u} c \frac{P_{i+1}^{n}-2 P_{i}^{n}+P_{i-1}^{n}}{h^{2}}=0 \\
& \frac{U_{i+1}^{n}-U_{i-1}^{n}}{2 h}-\frac{\tau_{u}}{\Delta t} \frac{U_{i+1}^{n}-U_{i+1}^{n-1}-U_{i-1}^{n}+U_{i-1}^{n-1}}{2 h}-\tau_{u} c \frac{U_{i+1}^{n}-2 U_{i}^{n}+U_{i-1}^{n}}{h^{2}} \\
& -\frac{\tau_{u}}{\rho} \frac{P_{i+1}^{n}-2 P_{i}^{n}+P_{i-1}^{n}}{h^{2}}=0
\end{aligned}
$$

\section{A.2. Stencil of Chorin's method}

This is the usual decoupled projection method, with the intermediate velocity eliminated through lumping of the velocity mass matrix

$$
\begin{aligned}
& \frac{\rho}{6 \Delta t}\left(U_{i-1}^{n}+4 U_{i}^{n}+U_{i+1}^{n}-U_{i-1}^{n-1}-4 U_{i}^{n-1}-U_{i+1}^{n-1}\right)+\rho c \frac{U_{i+1}^{n}-U_{i-1}^{n}}{2 h} \\
& -2 \mu \frac{U_{i+1}^{n}-2 U_{i}^{n}+U_{i-1}^{n}}{h^{2}}+\frac{P_{i+1}^{n}-P_{i-1}^{n}}{2 h}+c \Delta t \frac{P_{i+2}^{n}-2 P_{i}^{n}+P_{i-2}^{n}}{(2 h)^{2}} \\
& +\frac{\mu \Delta t}{\rho} \frac{P_{i-2}^{n}-2 P_{i-1}^{n}+2 P_{i+1}^{n}-P_{i+2}^{n}}{h^{3}}=0
\end{aligned}
$$


$\frac{U_{i+1}^{n}-U_{i-1}^{n}}{2 h}+\frac{\Delta t}{\rho}\left(\frac{P_{i+2}^{n}-2 P_{i}^{n}+P_{i-2}^{n}}{(2 h)^{2}}-\frac{P_{i+1}^{n}-2 P_{i}^{n}+P_{i-1}^{n}}{h^{2}}\right)=0$

\section{APPENDIX B}

Here we briefly describe the main commands used to obtain the results of Section 6 concerning von Neumann's analysis, with the help of the symbolic manipulator Mathematica 3.0. First, it is convenient to independently describe the method's stencil and the obtention of the discriminant matrix in an ASCII file containing the corresponding commands, to be read and executed later by Mathematica. As an example, a possible organization of this file for SPGP method with vanishing source term is (the variables' names have been kept as intuitive as possible)

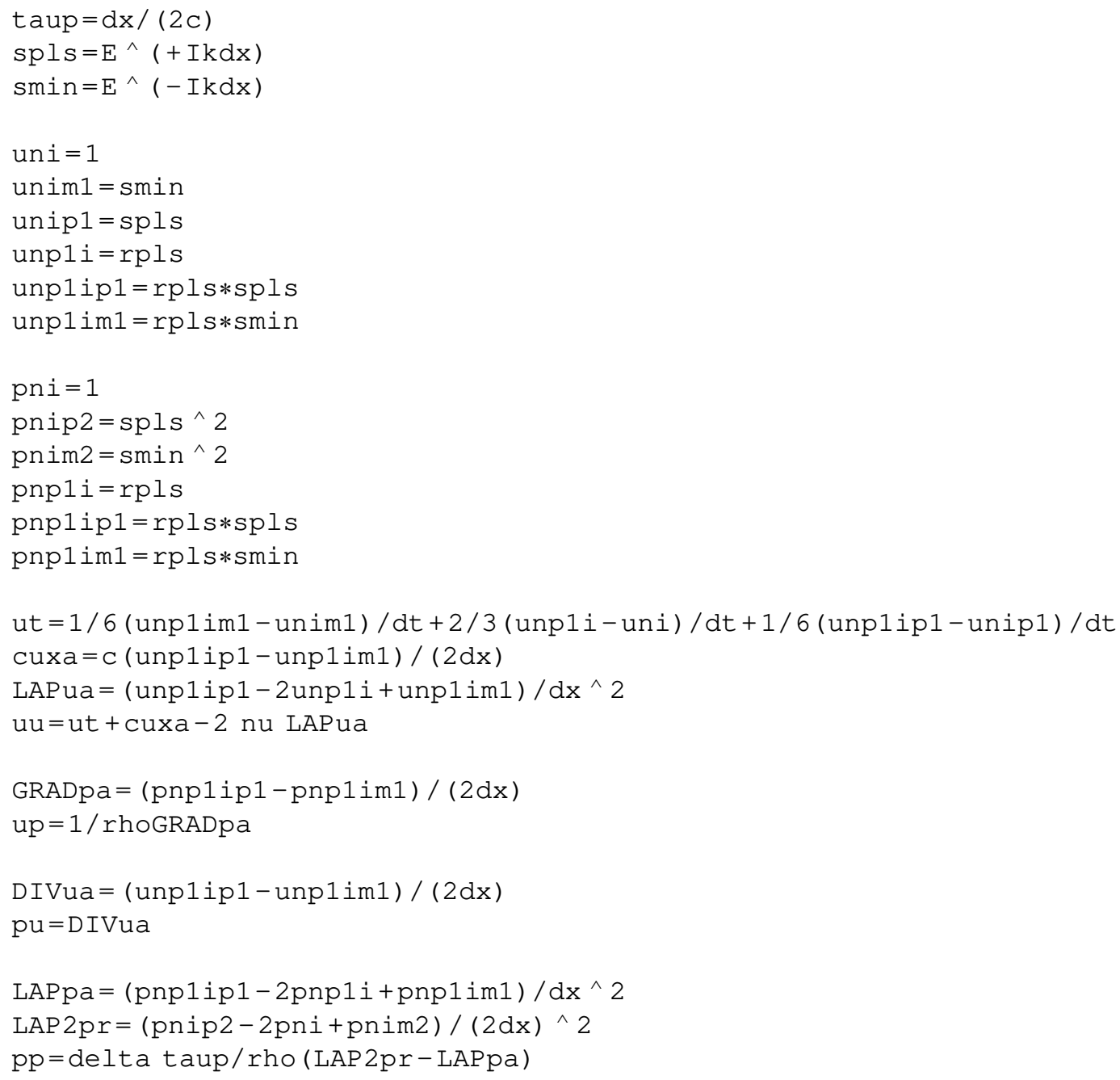


Let the name of this file be spgp.mat. Then, the essential commands for Mathematica's session defining all the necessary explicit functions are

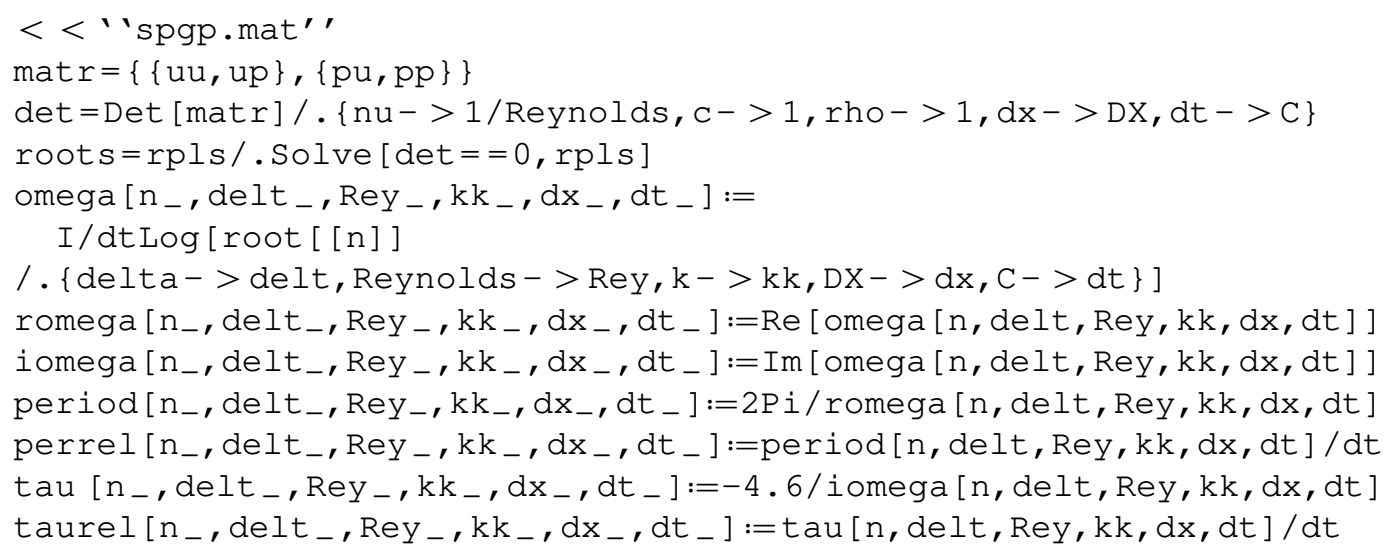

For the preceding commands, $n=1,2$ stands for root's number. 'omega' $(\omega)$ is defined by $r=\mathrm{e}^{-\mathrm{I} \omega \Delta t}$.

\section{REFERENCES}

1. Franca L, Frey S. Stabilized finite element methods: II. The incompressible Navier-Stokes equations. Computer Methods in Applied Mechanics and Engineering 1992; 99: 209-233.

2. Franca L, Hughes T, Stenberg R. Stabilized finite element methods. In Incompressible Computational Fluid Dynamics, Gunzburger M, Nicolaides R (eds). Cambridge University Press: Cambridge, 1993; 87-107.

3. Chorin AJ. Numerical solution of the Navier-Stokes equations. Mathematics of Computation 1968; 22: 745-762.

4. Temam R. Sur l'approximation de la solution des équations de Navier-Stokes par la méthode des pas fractionnaires (I). Archive of Rational Mechanics and Analysis 1969; 32: 135-153.

5. Colella P, Puckett EG. Modern numerical methods for fluid flow. http://patzek.berkeley.edu/PE/modern numerical_methods_for_flu.htm, 1994.

6. Daniels H, Peters A. Solving large incompressible time-dependent flow problems on scalable parallel systems. In Solution Techniques for Large-Scale CFD Problems, Habashi WG (ed.). Wiley: New York, 1995; 27-40.

7. Guermond J-L, Quartapelle L. Calculation of incompressible viscous flows by an unconditionally stable projection FEM. Journal of Computational Physics 1997; 132: 12-33.

8. Guermond J-L, Quartapelle L. On stability and convergence of projection methods based on pressure Poisson equation. International Journal for Numerical Methods in Fluids 1998; 26: 1039-1053.

9. Nonino C, Comini G. An equal-order velocity-pressure algorithm for incompressible thermal flows. Part 1: formulation. Numerical Heat Transfer 1997; B32: 1-15.

10. Nonino C, Comini G. An equal-order velocity-pressure algorithm for incompressible thermal flows. Part 2: validation. Numerical Heat Transfer 1997; B32: 17-35.

11. Simo JC, Armero F. Unconditional stability and long-term behavior of transient algorithms for the incompressible Navier-Stokes and Euler equations. Computer Methods in Applied Mechanics and Engineering 1994; 111: $111-154$.

12. Habashi W, Peeters M, Robichaud M, Nguyen V-N. A fully-coupled finite element algorithm, using direct and iterative solvers, for the incompressible Navier-Stokes equations. In Incompressible Computational Fluid Dynamics, Gunzburger M, Nicolaides R (eds). Cambridge University Press: Cambridge, 1993.

13. Zienkiewicz OC, Codina R. A general algorithm for compressible and incompressible flow-part I. The split, characteristic-based scheme. International Journal for Numerical Methods in Fluids 1995; 20: 869-885.

14. Codina R, Blasco J. A finite element formulation for the Stokes problem allowing equal velocity-pressure interpolation. Computer Methods in Applied Mechanics and Engineering 1997; 143: 373-391.

15. Codina R, Blasco J. Stabilized finite element method for the transient Navier-Stokes equations based on a pressure gradient projection. Computer Methods in Applied Mechanics and Engineering 2000; 182: 277-300. 
16. Codina R, Blasco J. Analysis of a finite element approximation of the stationary Navier-Stokes equations using equal velocity-pressure interpolation. Numerische Mathematik, in press.

17. Codina R. Stabilization of incompressibility and convection through orthogonal sub-scales in finite element methods. Computer Methods in Applied Mechanics and Engineering, in press.

18. Hughes T. Multiscale phenomena: Green's function, the Dirichlet-to-Neumann formulation, subgrid scale models, bubbles and the origins of stabilized formulations. Computer Methods in Applied Mechanics and Engineering 1995; 127: $387-401$

19. Lew A. El método de elemento finitos en entornos computacionales de alta performance. Master's thesis, Instituto Balseiro, 1998.

20. Codina R. A stabilized finite element method for generalized stationary incompressible flows. Computer Methods in Applied Mechanics and Engineering, in press.

21. Idelsohn S, Storti M, Nigro N. Stability analysis of mixed finite element formulations with special mention of equal-order interpolations. International Journal for Numerical Methods in Fluids 1995; 20: 1003-1022.

22. Wolfram S. Mathematica (2nd edn). Addison-Wesley: Reading, MA, 1991.

23. Bouard R, Coutanceau M. The early stage of development of the wake behind an impulsively started cylinder for $40<R e<10^{4}$. Journal of Fluid Mechanics 1980; 101: 583-607.

24. Tanahashi T, Okanaga H. GSMAC finite element method for unsteady incompressible Navier-Stokes equations at high Reynolds numbers. International Journal for Numerical Methods in Fluids 1990; 11: 479-499.

25. Gresho P. Incompressible fluid dynamics: some fundamental formulation issues. Annual Review of Fluid Mechanics 1991; 23: 423-453. 